WATER QUALITY OF RHODE ISLAND STREAMS

By John C. Briggs and Jeffrey S. Feiffer

U.S. GEOLOGICAL SURVEY

Water-Resources Investigations Report 84-4367

Prepared in cooperation with the

STATE OF RHODE ISLAND

DEPARTMENT OF ENVIRONMENTAL MANAGEMENT

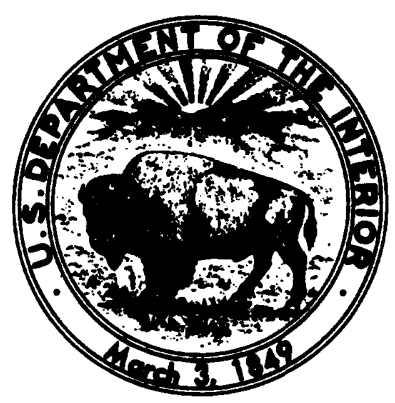

Boston, Massachusetts 


\section{UNITED STATES DEPARTMENT OF THE INTERIOR \\ DONALD PAUL HODEL, Secretary \\ GEOLOGICAL SURVEY \\ Dallas L. Peck, Director}

For additional information write to:

U.S. Geological Survey

150 Causeway Street, Suite 1309

Boston, MA 02114-1384
Copies of this report can be purchased from:

Open-File Services Section

Western Distribution Branch

U.S. Geological Survey

Box 25425, Federal Center

Denver, CO 80225

Telephone: (303) 236-7476 
Abstract --- - -

Introduction -.-

Purpose and scope -

Description of the stream stations

Methods of study

Water quality of streams

Major inorganic chemicals - 7

Calcium, magnesium, and hardness - 7

Sodium --.-_- 7

Chloride and sulfate - 7

Fluoride --1-0-10 7

Major nutrients -

Nitrogen - 8

Phosphorus - 9

Bacteria--

Trace elements - 19

Organic compounds - 27

Constituents in bottom materials - 27

Trends in water quality - 46

Method -- 47

Results --

Summary --

References cited -_- 50

\section{ILLUSTRATIONS}

Figure 1. Map showing location of study area and sampling stations --

2. Bar chart showing mean streamflow by station, 1979-83

3. Bar chart showing mean total nitrogen by station, 1979-83 - 10

4. Bar chart showing mean total phosphorus by station, 1979-83-11

5. Bar chart showing mean fecal coliform bacteria by station, 1979-83 - 12

6. Bar chart showing maximum fecal coliform bacteria by station, 1979-83 12 


\section{TABLES}

Table 1. Water quality sampling stations within the study area

2. Characteristics and constituents currently measured at Rhode Island stations - 5

3. Summary of measurements of common constituents, nutrients, bacteria, and field measurements for each station -... 13

4. Summary of measurements of trace elements for each station

5. Selected criteria and the maximum concentration for selected trace elements for each station - 26

6. Summary of measurements of organic compounds for each station -..-- 28

7. Summary of measurements of constituents in the bottom material for each station 34

8. Trend results for each station 48

\section{CONVERSION FACTORS}

The following factors can be used to convert inch-pound units to International System of Units (SI).

\section{Length}

foot (ft)

mile (mi)

0.3048

1.609

$\underline{\text { Area }}$

square mile $\left(\mathrm{mi}^{2}\right)$ meter (m)

kilometer $(\mathrm{km})$

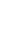
3 20 6 8 . 8 


\title{
WATER QUALITY OF RHODE ISLAND STREAMS
}

\author{
by John C. Briggs and Jeffrey S. Feiffer
}

\section{ABSTRACT}

Water-quality data collected from November 1978 through September 1983 at five stations within Rhode Island and one in Massachusetts show that concentrations of the common constituents were low. Mean water hardness at all sites was in the "soft" category. Sodium concentrations were less than 20 milligrams per liter at two sites and less than 35 milligrams per liter at the other sites. Mean nitrogen values for the two Blackstone River sites were in the range that could cause undesirable growths of aquatic plants. Mean phosphorus values exceeded the recommended limits for protection of aquatic life at four sites.

Trace-element concentrations in the water were generally low. Those trace elements which were found in concentrations near or exceeding any standard or criterion include cadmium, chromium, lead, iron, and manganese. High concentrations of several trace elements were found in the bottom materials at several sites. The bottom materials also contained pesticides and organic chemicals including aldrin, chlordane, DDD, DDE, DDT, dieldren, endosulfan, endrin, heptachlor, mirex, and PCB.

Results of trend analysis of total phosphorus, total nitrogen, and specific conductance show a downward trend in phosphorus at two sites; an upward trend in nitrogen at one site; and one downward trend and one upward trend in specific conductance.

\section{INTRODUCTION}

The State of Rhode Island is required by several Federal laws to monitor, compile, and analyze data on the quality of streams. For the State to meet these requirements, water-quality data are needed for preparing comprehensive water-pollution control plans, developing information on the causes and effects of increases or decreases in pollution, determining water quality of major rivers on a regular basis and appraising long-term water-quality trends, and evaluating the effects of surface-water quality management strategies.

The U.S. Geological Survey requires information on water quality to accomplish the Agency's mission to appraise the quantity, quality, and use of the Nation's water. Two of the Survey's NASQAN (National Stream Quality Accounting Network) stations are located on rivers which are located in or flow into Rhode Island. These stations are the Pawcatuck River at Westerly, R.I., and the Blackstone River at Millville, Mass. However, additional sites were needed to give better spatial coverage of the State and to supplement the information that is being collected at the NASQAN sites. To address these problems, a program to collect water quality data from four additional Rhode Island streams, plus additional data to supplement the two NASQAN stations, was initiated in October 1978 in cooperation with the Rhode Island Department of Environmental Management. 


\section{Purpose and Scope}

Specific objectives of the project are to (1) determine the existing chemical, physical, and biological quality of Rhode Island's major streams and (2) accumulate a data base, by periodic water-quality measurements, from which changes in stream quality can be assessed.

The purpose of this report is to summarize the data that were collected for the stations from November 1978 through September 1983, the end of the 1983 water year. Data from the stations in the study have been published on a water year (October through September) basis, in the series of publications, "Water Resources Data Massachusetts and Rhode Island Water Year (year)." Additional earlier data are included in the summary. This report includes an assessment of temporal trends in selected water-quality constituents.

\section{Description of Stream Stations}

Figure 1 shows the location of the six water-quality stations chosen for the study. Table 1 lists the station number, name, period of record, location, and any remarks about the site. Five of the sites are located within the State of Rhode Island while the sixth, Blackstone River at Millville, Mass., located just upstream from the Massachusetts-Rhode Island border, provides information on the water quality of the Blackstone River as it enters Rhode Island. All six sites are located downstream of regulated stream reaches. The Pawtuxet River is heavily regulated by the Scituate Reservoir, by the Flat River Reservoir, and by powerdams. The least regulated of the rivers is the Branch River at Forestdale whose upstream dams are not being actively used for power generation.

Two sites, Blackstone River at Millville, Mass., and Pawcatuck River at Westerly, are NASQAN sites. NASQAN, the National Stream Quality Accounting Network, is a set of over 500 stations nationwide at which a large number of water-quality characteristics of rivers are measured regularly. The major objectives of the NASQAN program are to: (1) Account for the quantity and quality of water moving within and from the United States; (2) depict the areal variablility of stream quality; (3) depict the temporal variability of stream quality; and (4) detect long-term trends in stream quality.

\section{METHODS OF STUDY}

Characteristics and constituents which are currently measured as part of the Rhode Island water-quality program are listed in table 2. Water samples are collected monthly for analysis of major nitrogen and phosphorus species, bacteria, and selected physical constituents and twice-yearly for analyses of common chemical constituents, trace elements, and organic compounds. Once yearly, bottom materials are examined for organic constituents.

Water samples are collected using fluvial-sediment sampling techniques described by Guy and Norman (1970). Use of these techniques ensures that samples collected for analysis of total chemical constituents contain a representative subsample of both the dissolved and suspended material passing through the stream cross-section at the time of sampling. The term "total" used with a constituent means that the sample consists of a water-sediment mixture and that the analytical method determines all of the constituent in the sample. The term "total, recoverable" is the amount of a given constituent in solution after a repesentative water-suspended sediment sample has been digested by a method (usually using a dilute acid solution) that results in dissolution of only readily soluble substances. Complete dissolution of all particulate matter is not achieved by the digestion treatment and thus the determination represents something less than the "total" a mount. A "dissolved" constituent refers to that material in a representative water sample which passes through a 0.45 -micrometer membrane filter. This is a convenient operational definition of a dissolved constituent used by Federal agencies and many State agencies that collect water samples. 


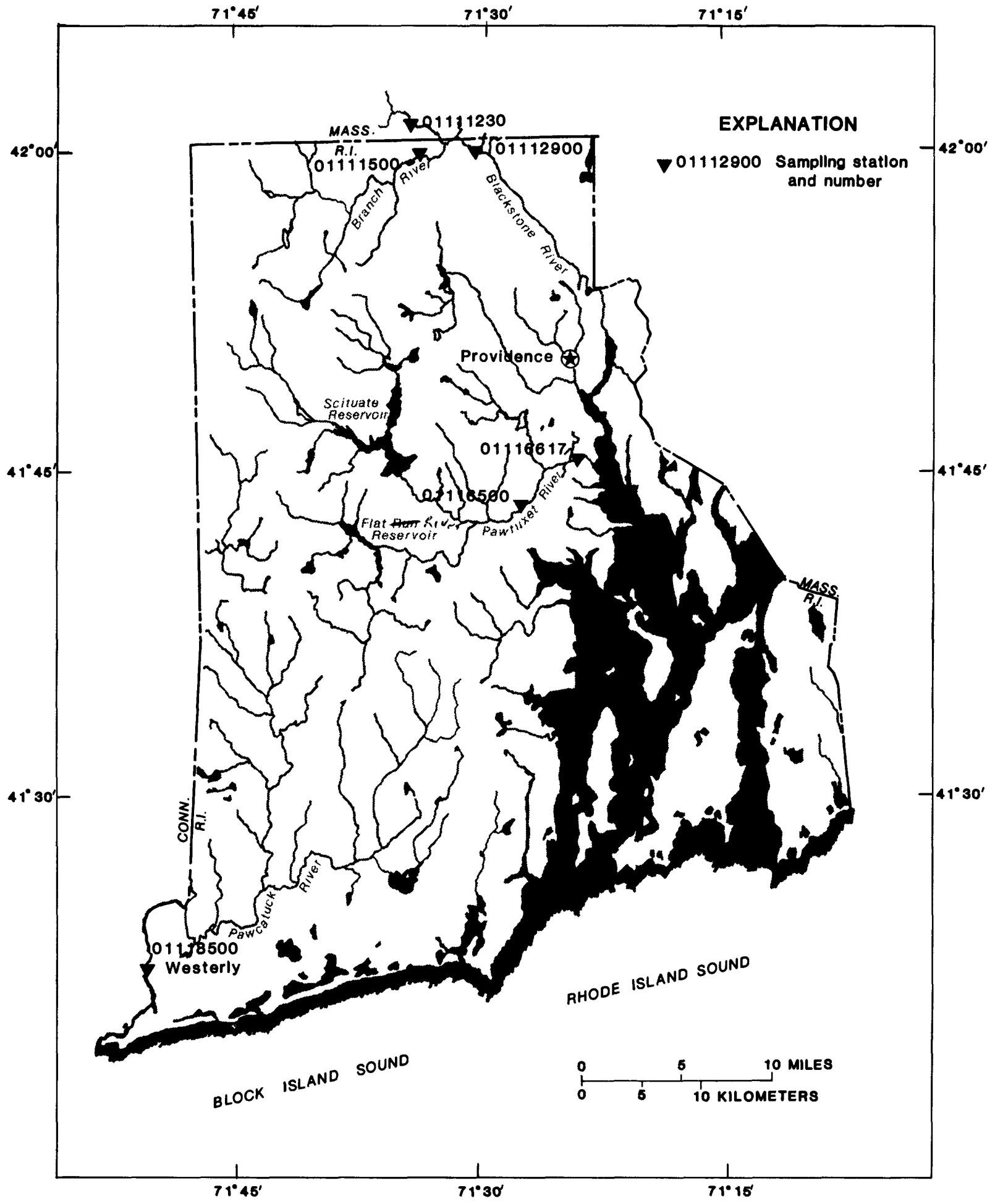

Figure 1.--Location of study area and sampling stations 
Table 1.-Water quality sampling sites within the study area

Station number, station name, and period of record
Station location and remarks

01111230

Blackstone River at Millville Mass.

November 1978-September 1983

Samples: 1971 and 1973 included.

01111500

Branch River at

Forestdale, R.I.

May 1975-September 1983

Samples: 1953-58, 1965-69, and 1971-72 included.

01112900

Blackstone River at Manville, R.I.

November 1978-September 1983

Samples: 1970 included.

01116500

Pawtuxet River at

Cranston, R.I.

May 1978-September 1983

Samples: 1953-58, 1961-73, and 1975-77 included.

\section{7}

Pawtuxet River at

Pawtuxet, R.I.

November 1978-September 1983

\section{0}

Pawcatuck River at

Westerly, R.I.

April 1976-September 1983
Latitude 42001'16", longitude 71034'04", Worcester County, 400 feet above railroad bridge, 0.8 mile southeast of Millville, 1 mile upstream from the Rhode Island-Massachusetts border, and 1.4 miles upstream from the Branch River, drainage area $277 \mathrm{mi}^{2}$. Flow regulated by powerdams, by West Hill Reservoir, and by other upstream reservoirs.

Latitude 41059'47", longitude 71033'47", Providence County, 400 feet downstream from the milldam at Forestdale, 1 mile east of Slatersville, and $1.6 \mathrm{mi}$ upstream from the mouth, drainage area $91.2 \mathrm{mi}^{2}$. No active regulation by milldams upstream.

Latitude 41058'18", longitude 71028'14", Providence County, at milldam at Manville, and 400 feet upstream from Manville Road Bridge. Flow regulated by powerdams, by West Hill Reservoir, and by other upstream reservoirs.

Latitude 41045'03", longitude 71026'44", Providence County, at Cranston, $\mathbf{0 . 7}$ mile upstream from Pocasset River, 1.4 miles upstream from the I-95 bridge, drainage area $200 \mathrm{mi}^{2}$. Flow regulated by powerplants and the Scituate, Flat River, and other reservoirs.

Latitude 41045'51", longitude 71023'45", Providence County, 1,300 feet upstream from the Narragansett Parkway Bridge at Pawtuxet, and 1,500 feet upstream stream from the mouth. Flow regulated by powerplants and the Scituate, Flat River, and other reservoirs.

Latitude 41023'01", longitude 71050'01", Washington County, at Westerly, 2.1 miles downstream from the Shunock River, drainage area $295 \mathrm{mi}^{2}$. Some regulation from mill dams. 
Table 2.-Characteristics and constituents currently measured at Rhode Island stations

\section{MEASURED MONTHLY}

Field determinations

Discharge

Water temperature

Specific conductance

$\mathrm{pH}$

Dissolved oxygen

Biological characteristics

Fecal coliform bacteria

Fecal streptococci bacteria

Other

5 day biological oxygen demand (BOD)
Major nutrients

Nitrogen

Total nitrogen

Total organic nitrogen

Total ammonia nitrogen

Total nitrite nitrogen

Total nitrate nitrogen

Phosphorus

Total phosphorus

Total orthophosphate

MEASURED TWICE YEARLY DURING

LOW AND HIGH FLOW

Common constituents

Dissolved calcium

Dissolved magnesium

Dissolved sodium

Dissolved potassium

Dissolved chloride

Dissolved sulfate

Alkalinity

Other

Color

Chemical oxygen demand

Total ROE (residue on evaporation) at $105^{\circ} \mathrm{C}$

Suspended ROE at $105^{\circ} \mathrm{C}$

Phenols

Total oil and grease
Trace elements

Total alluminum

Total arsenic

Total boron

Total cadmium

Total chromium

Total copper

Total iron

Total lead

Total manganese

Total mercury

Total molybdinum

Total nickel

Total selenium

Total silver

Total zine
Organic compounds

Total aldrin

Total chlordane

Total DDD

Total DDE

Total DDT

Total dieldrin

Total endosulfan

Total endrin

Total PCB

Total PCN

Total heptachlor epoxide

Total heptachlor

Total lindane

Total methoxychlor

Total myrex

Total perthane

Total toxaphene

\section{MEASURED YEARLY DURING LOW FLOW}

Organic compounds in stream bottom material

Total aldrin

Total DDE

Total endosulfan

Total PCN

Total lindane

Total perthane
Total chlordane

Total DDT

Total endrin

Total heptachlor epoxide

Total mirex

Total toxaphene
Total DDD

Total dieldrin

Total PCB

Total hep tachlor

Total methoxychlor 
Stream-discharge values are either from measurements made at the time of sample collection or from a stage-discharge rating (Rantz, 1982). Specific conductance and $\mathrm{pH}$ were measured using methods described by Wood (1976), and dissolved oxygen concentration was measured using a dissolved-oxygen meter and techniques described in Skougstad and others (1979). samples collected for laboratory analyses were preserved in the field and immediately shipped to the Survey laboratory in Atlanta, Georgia. Sample preservation and analytical methods are described in Skougstad and others (1979), Greeson and others (1977), Greeson (1979), and Wershaw and others (1983).

Certain constituents are often not found in a sample of water from a stream. For example, the concentration of trace elements, organic compounds, or bacteria may be below the detection limit of the analytical procedure. Normally these results are reported and published as "less than" the detection limit of the analytical procedure used for the determination. Results of individual analyses which are published in the annual data report will of ten show a "less than" concentration for a particular constituent. Detection limits for any particular constituent are given in the references to analytical procedures given in the preceding paragraph. When summarizing a number of analyses using statistical techniques, these "less than" values must be taken into account. For this report, all "less than" values have been converted to zero. All tables and figures which show a zero value should be interpreted as below the detection limit of the analyses.

Results from determination of the number of bacteria in a sample of water may occasionally be reported as "greater than" a value. For this report, those values were treated as an absolute value. In the summary tables and in figures showing maximum values for bacteria counts, the actual value may have been greater than the maximum shown. This information is available in the individual analyses.

\section{WATER QUALITY OF ST REAMS}

There is no clear, simple, quantitative way to describe the term "water quality." In practice, the quality of a water resource is determined by various measurements of physical, chemical, and biological characteristics. Results of the measurements can be compared with water-quality standards or criteria in order to judge the suitability of the water. There are several different sets of criteria, depending upon the intended use of the water. Water that meets the criteria for one particular use will not necessarily meet the criteria for other uses.

Discussions in the subsections that follow deal with the results of measurements of selected water-quality characteristics, and relate the results to some of the criteria for certain water uses. Among the commonly cited standards and criteria are: Water Quality Criteria 1972 (National Academy of Sciences and National Academy of Engineering, 1972); Quality Criteria for Water (U.S. Environmental Protection Agency, 1976); National Interim Primary and Secondary Drinking Water Regulations (U.S. Environmental Protection Agency, 1975, 1977, 1980); and recommended criteria proposed in miscellaneous articles and reports. In many cases, more stringent criteria are cited than would normally be imposed on a stream. For example, drinking water criteria may be cited even though the stream is not used as a drinking water source. The criteria are used to give the reader benchmarks with which to compare concentrations of constituents in the stream.

Additional confusion occurs in trying to decide if drinking water criteria should be applied to "total", "total recoverable", or "dissolved" constituents. Most of the criteria for domestic water supply apply to water which is delivered to the free flowing outlet of the ultimate user of a public water system (U.S. Environmental Protection Agency, 1975). Water from a stream used as a drinking water source may have been filtered or otherwise treated to remove particulate matter. While this water may not meet the strict definition of dissolved given above, the analyses of the water will probably be closer to a "dissolved" analyses of the source water rather than an analyses for "total" or 
"total recoverable" constituents unless the water has had chemical treatment. Chemical treatment may result in a water whose analytical results will be quite different from even the dissolved analyses of the untreated water. In this report, drinking water criteria may be compared with "total", "total recoverable", or "dissolved" constituents. Again, comparison with drinking water criteria give the reader a benchmark with which to compare concentrations of constituents within the stream.

\section{Major Inorganic Chemicals}

The major inorganic chemicals dissolved in water often are termed "common constituents" because these substances generally dominate the total mass of dissolved material in water and have been the subject of most chemical analyses performed by the Geological Survey in the past. A summary of data on major inorganic chemicals measured at each of the Rhode Island stations is included in table 3. Also included in table 3 are physical measurements of the water such as specific conductance and temperature, biological measurements, and measurements of streamflow and suspended sediment.

\section{Calcium, Magnesium, and Hardness}

The presence of calcium and magnesium along with other metallic elements such as iron, strontium, and manganese, cause hardness in water. Many industrial and domestic water users are concerned about hardness. Hard water requires more soap and synthetic detergents for home laundry and washing than does soft water, and contributes to scaling in boilers and industrial equipment. In this report, hardness is reported as an equivalent mass of calcium carbonate, $\mathrm{CaCO}_{3}$. General guidelines for classification of waters are: 0 to $60 \mathrm{mg} / \mathrm{L}$ as $\mathrm{CaCO}_{3}$ is classified as soft; 61 to $120 \mathrm{mg} / \mathrm{L}$ as moderately hard; 121 to $180 \mathrm{mg} / \mathrm{L}$ as hard; and more than $180 \mathrm{mg} / \mathrm{L}$ as very hard (Durfor and Becker, 1964).

Mean concentrations of calcium, magnesium, and hardness, were low at all the Rhode Island sites. Mean hardness was in the soft water category at all sites, and maximum measured hardness was in the moderately hard range at only two sites; Pawtuxet River at Cranston and Pawtuxet River at Pawtuxet.

\section{Sodium}

The most restrictive drinking water criterion for sodium is $20 \mathrm{mg} / \mathrm{L}$. This criterion was set to protect individuals who may be on "very resticted sodium diets." Mean sodium concentration at Branch River at Forestdale and Pawcatuck River at Westerly meet this criterion. Mean concentrations at the other four sites range from 26 to $33 \mathrm{mg} / \mathrm{L}$. The highest measured concentration was $69 \mathrm{mg} / \mathrm{L}$ at Pawtuxet River at Pawtuxet.

\section{Chloride and Sulfate}

The presence of chloride can adversely affect taste in drinking water, and can cause corrosion and other problems in industrial water supplies. A large concentration of sulfate in drinking water is undesirable because of its laxative effects. To prevent these effects, a recommended limit of $250 \mathrm{mg} / \mathrm{L}$ for chlorides and sulfates in domestic water supplies was set by the U.S. Environmental Protection Agency (1977). The highest concentration of chloride and sulfate measured in the study area were 82 and $85 \mathrm{mg} / \mathrm{L}$, respectively. Mean chloride concentrations were greatest in the Pawtuxet River at Cranston and the Blackstone River at Millville, Mass. The mean sulfate concentration was greatest in the Pawtuxet River at Pawtuxet.

\section{Fluoride}

A concentration up to about $1 \mathrm{mg} / \mathrm{L}$ of fluoride is considered to have a beneficial health effect in drinking water through the reduction in the incidence of tooth decay. However, large concentrations of fluoride can produce dental fluorosis-mottling and chipping of tooth enamel. While there are relatively few analyses of fluoride at the six sites, the concentrations were low. Measured concentrations varied from 0.0 to $0.5 \mathrm{mg} / \mathrm{L}$. 


\section{Major Nutrients}

The major species of nitrogen and phosphorus are commonly found at low concentrations in natural waters. Significant concentrations of nitrogen and phosphorus in streams are usually the result of the addition of municipal or industrial wastewater or runoff from agricultural areas. This nutrient enrichment is undesirable because it may lead to algal blooms which subsequently can reduce the dissolved oxygen concentrations.

Frequently, there is confusion caused by the units used in the reporting of concentration of nitrogen and phosphorus. Some investigators report the concentrations of ions in mass per unit volume, such as nitrogen as $\mathrm{NO}_{3}$ (nitrate) and phophorus as $\mathrm{PO}_{4}$ (phosphate). Other investigators report only the mass of nitrogen or phosphorus, such as nitrogen as $\mathbf{N}$ and phophorus as $\mathbf{P}$. The latter method is used throughout this report and gives results considerably different from the former. For example, $1.0 \mathrm{mg} / \mathrm{L}$ of nitrate nitrogen as $\mathrm{N}$, is equivalent to $4.4 \mathrm{mg} / \mathrm{L}$ of nitrate, $\mathrm{NO}_{3}$. Differences may also be found in the schemes for reporting nitrogen and phosphorus as dissolved, suspended, or total. Data in this report represent both "dissolved" concentrations, which include the portions in solution, and "total" concentrations, which include the dissolved plus the portions that are associated with suspended material.

\section{Nitrogen}

The most common nitrogen forms found in water are nitrate, nitrite, ammonia, and organic nitrogen. For a particular analysis, results can be summed for these constituents and reported as "nitrogen." Organic nitrogen is nitrogen which is included within complex carbon-containing molecules formed by plants and animals. Waste material from living organisms, as well as their remains after death, are decomposed by bacterial action releasing nitrogen compounds. Organic compounds containing nitrogen are further broken down by bacteria into ammonia. Ammonia is converted by other bacteria to nitrite and then rapidly into nitrate in the presence of oxygen.

Criteria for nitrogen in water are generally based on the nitrate concentration. The U.S. Environmental Protection Agency (1976) specifies $10 \mathrm{mg} / \mathrm{L}$ as the maximum allowable concentration of nitrate nitrogen for drinking water-supplies to provide human health protection. Criteria vary on concentrations of nitrate for the protection of aquatic life; that is, levels which will not lead to nuisance growths of algae and other aquatic plants. The U.S. Council on Environmental Quality (1975) used a maximum concentration of $0.6 \mathrm{mg} / \mathrm{L}$ of nitrate nitrogen as a "benchmark" level for aquatic life protection, suggesting that higher levels are indicative of undesirable eutrophication. Other criteria for nitrate nitrogen established across the country to limit eutrophication vary from 0.10 $\mathrm{mg} / \mathrm{L}$ in pristine waters to $3 \mathrm{mg} / \mathrm{L}$ in less sensitive waters. A study of 365 sampling points on major rivers within the United States showed that nitrate nitrogen levels were below $1.0 \mathrm{mg} / \mathrm{L}$ at 85 percent of the sites and below $0.5 \mathrm{mg} / \mathrm{L}$ at 65 percent of the sites (Briggs and Ficke, 1978).

Mean total nitrate plus nitrite concentrations were lowest in the Branch River at Forestdale and in the Pawcatuck River at Westerly, with values of 0.30 and $0.39 \mathrm{mg} / \mathrm{L}$, respectively. Nitrate plus nitrite was used since the nitrite concentration is quite small compared to the nitrate concentration. Highest mean total nitrate plus nitrite values were in the Blackstone River at Millville, Mass. and in the Blackstone River at Manville with 1.4 and $1.1 \mathrm{mg} / \mathrm{L}$, respectively. While these values are lower than the drinking water standard, they are in a range that could cause growth of excessive quantities of aquatic plants if the water were impounded in a lake and if the supply of other essential nutrients was sufficent.

Total nitrogen concentrations were lowest again in the Branch River at Forestdale and in the Pawcatuck River at Westerly, with mean values of 0.93 and $0.88 \mathrm{mg} / \mathrm{L}$, respectively. Highest mean concentrations were in the Blackstone River at Millville, Mass., and in the Pawtuxet River at Pawtuxet with mean concentrations of 3.2 and $2.7 \mathrm{mg} / \mathrm{L}$. 
The variability of the nitrogen concentrations in different years may be partially related to streamflow. Figure 2 shows the mean streamflow in cubic feet per second by year for the years 1979 through 1983 . Figure 3 shows the annual mean total nitrogen concentration for 1979 through 1983 for the six sites. In general, the years with lower mean streamflow values had higher mean nitrogen values. Mean streamflow was lowest in 1981 at all of the sites. Mean concentrations of nitrogen were highest in 1981 at the Blackstone River at Manville (01112900), the Pawtuxet River at Cranston (01116500), the Pawtuxet River at Pawtuxet (01116617), and the Pawcatuck River at Westerly (01118500).

\section{Phosphorus}

Phosphorous data are reported as "total phosphorus as $\mathrm{P}$ " and "total or thophosphate phorphorus as P". Total phophorus includes all forms of phosphorus such as soluble or thophosphate, soluble hydrolyzable phophorus, soluble organic phosphorus and phosphorus associated with collodial material and both inorganic and organic suspended material. Even though orthophophate phosphorus is the only phosphorus form that is readily available as a plant nutrient, the total amount of phosphorus is an important environmental measurement because phophorus may be converted by biological or chemical means into or thophosphate phosphorus.

The U.S. Environmental Protection Agency (1976) proposed a phosphorus criterion of $0.05 \mathrm{mg} / \mathrm{L}$ for any stream at the point where it enters a lake or reservoir and 0.025 $\mathrm{mg} / \mathrm{L}$ within the lake or reservoir. This criterion is established to prevent or control nuisance aquatic-plant growth. The U.S. Council on Environmental Quality (1976, p. 27) has suggested maximum concentrations of phophorus of $0.1 \mathrm{mg} / \mathrm{L}$ for "aquatic life protection."

Orthophosphate phosphorous concentrations were lowest at the Pawcatuck River at Westerly and at the Branch River at Forestdale with mean values of $0.02 \mathrm{mg} / \mathrm{L}$ at both sites and maximum values of 0.09 and $0.26 \mathrm{mg} / \mathrm{L}$, respectively. Total phosphorus concentrations were lowest at the Branch River at Forestdale and the Pawcatuck River at Westerly with respective mean concentrations of 0.04 and $0.05 \mathrm{mg} / \mathrm{L}$. Highest mean total phosphorus and mean orthophosphate phosphorus concentrations was at the Pawtuxet River at Pawtuxet.

Figure 4 shows the annual mean total phorsphorus values for the six stations for the years 1979 through 1983 . The concentrations varied from year to year, generally with the highest concentrations in 1981 when streamflow was lowest. Only the Branch River at Forestdale (01111500) and the Pawcatuck River at Westerly (01118500) met either the 0.05 or the $0.1 \mathrm{mg} / \mathrm{L}$ criteria. The other four sites had phophorus concentrations which exceeded the criterion.

\section{Bacteria}

There are many species of bacteria in natural waters; some are pathogenic, or disease causing, but fortunately most are harmless. People, of course, are concerned mainly about those that present threats of disease.

It is impossible to monitor for all forms of pathogens. Instead, knowing that certain disease-producing organisms move from person to person through the water, the waters are examined for signs that these organisms may be present. Classically, this has been done by monitoring for "indicator" organisms in the so-called coliform bacterial group, and more recently in the fecal streptococci group. The logic of the scheme is that (1) indicator bacteria show the presence of fecal contamination, and (2) contaminated waters are likely to contain pathogens in numbers proportional to the numbers of indicator bacteria. 


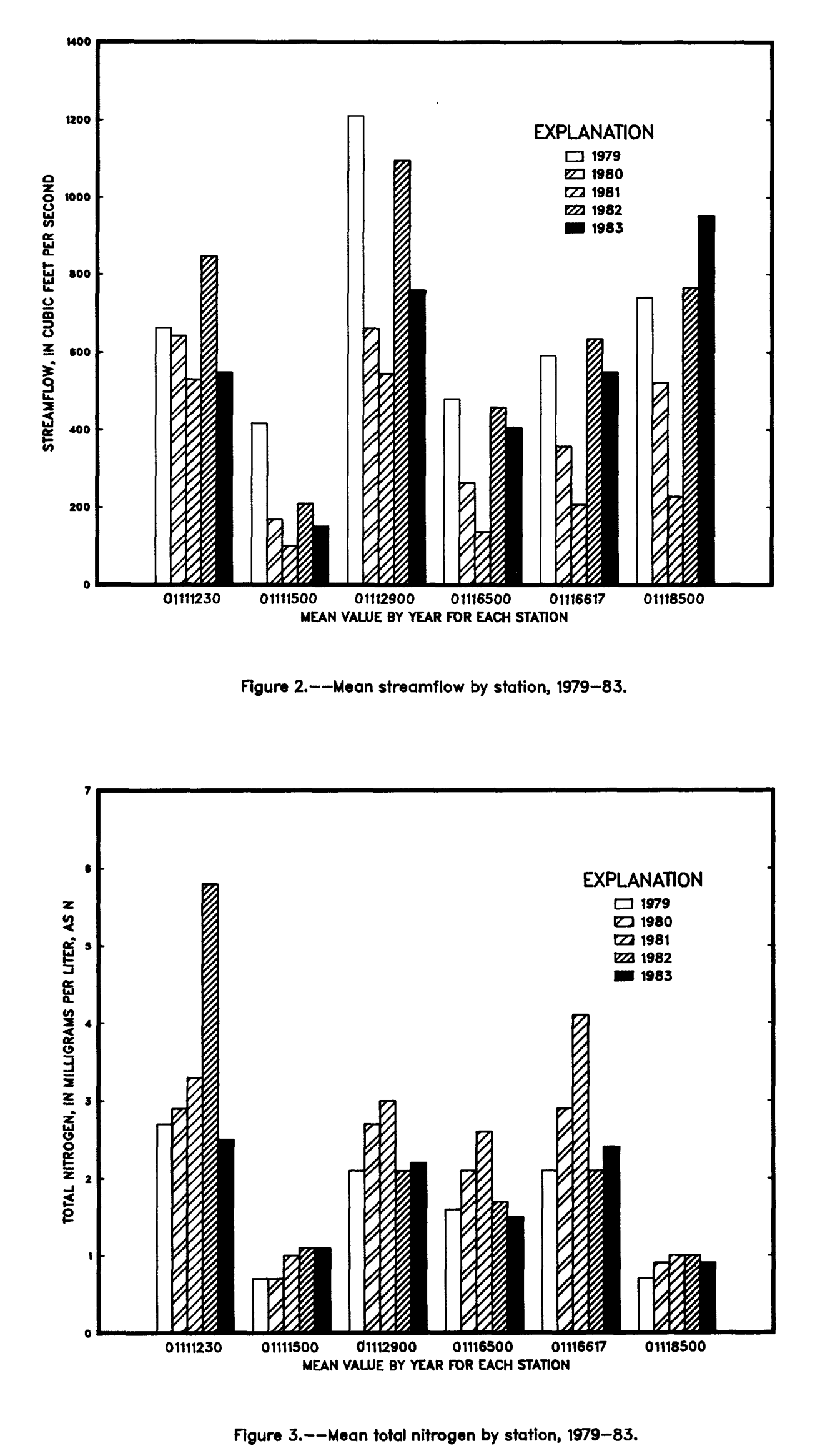




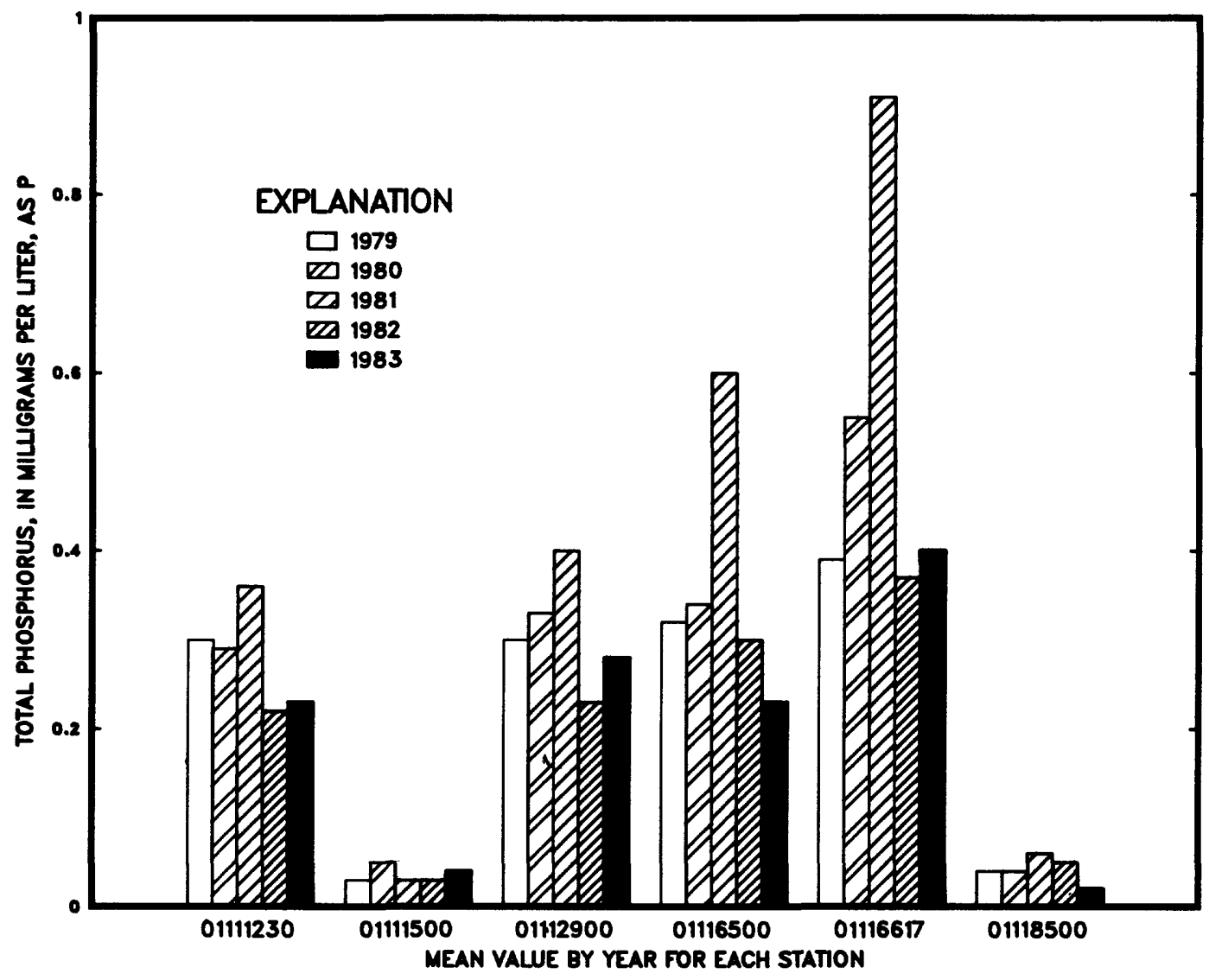

Figure 4.--Mean total phosphorus by station, 1979-83.

At the six stations studied, bacteria samples were collected to determine numbers of: (1) Fecal coliform bacteria, those members of the coliform group found in the feces of various warmblooded animals, and (2) fecal streptococci bacteria which are also found in the intestines of warmblooded animals. Most standards and criteria of water quality are written in terms of fecal coliform bacteria. For body contact sports such as swimming, the U.S. Environmental Protection Agency (1976) set a criterion that the fecal coliform bacteria count should not exceed a log mean (geometric mean) of 200 per $100 \mathrm{ml}$ of sample based on a minimum of five samples taken over a 30-day period. In addition, not more than 10 percent of the samples taken during the period should exceed 400 per $100 \mathrm{ml}$. Water Quality Criteria 1972 (National Academy of Sciences and National Academy of Engineering, 1972) recommended that raw waters used as a source for public supply contain not more than 2,000 colonies of fecal coliform bacteria per $100 \mathrm{ml}$.

Figures 5 and 6 show the arithmetic mean and the observed maximum number of fecal coliform bacteria per $100 \mathrm{ml}$ of sample for the most recent years at the six sites. As shown in the figures, none of the sites met the body-contact criterion. However, samples collected as part of this study were not collected in sufficient quantities to meet the sampling frequency requirement of the criterion but the numbers are indicative that the water may not meet the criterion. Each of the two sites with the lowest number of counts, the Pawtuxet River at Cranston (01116500) and the Pawtuxet River at Pawtuxet (01116617), is located downstream from a wastewater-treatment plant. Their lower counts may reflect the effect of chlorinated effluent from the plants. 


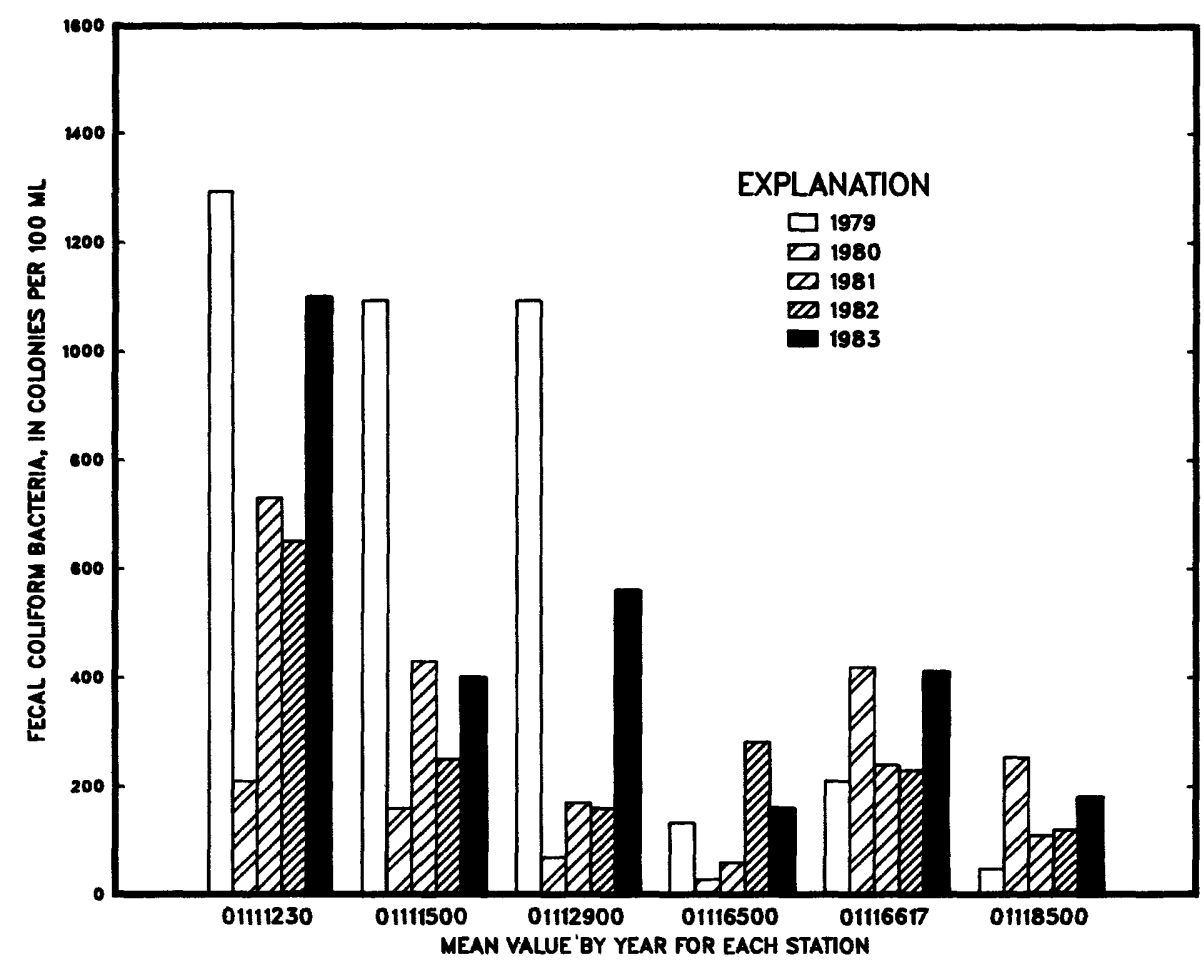

Figure 5. - Mean fecal coliform bacteria by station, 1979-83.

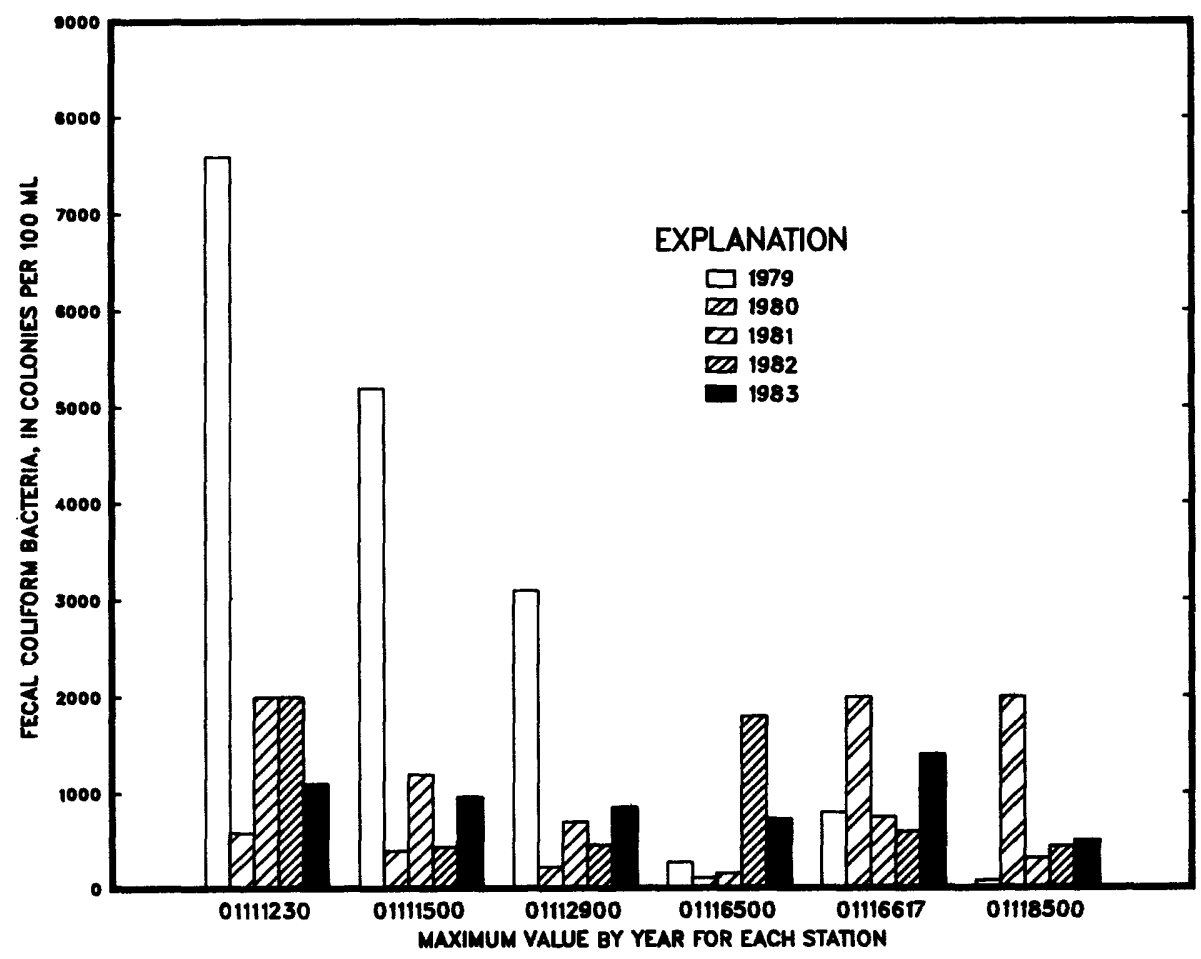

Figure 6. - Maximum fecal coliform by station, 1979-83. 
Table 3.-Summary of measurements of common constituents, nutrients, bacteria, and field measurements for each station

(Analyses are in milligrams per liter except as indicated.)

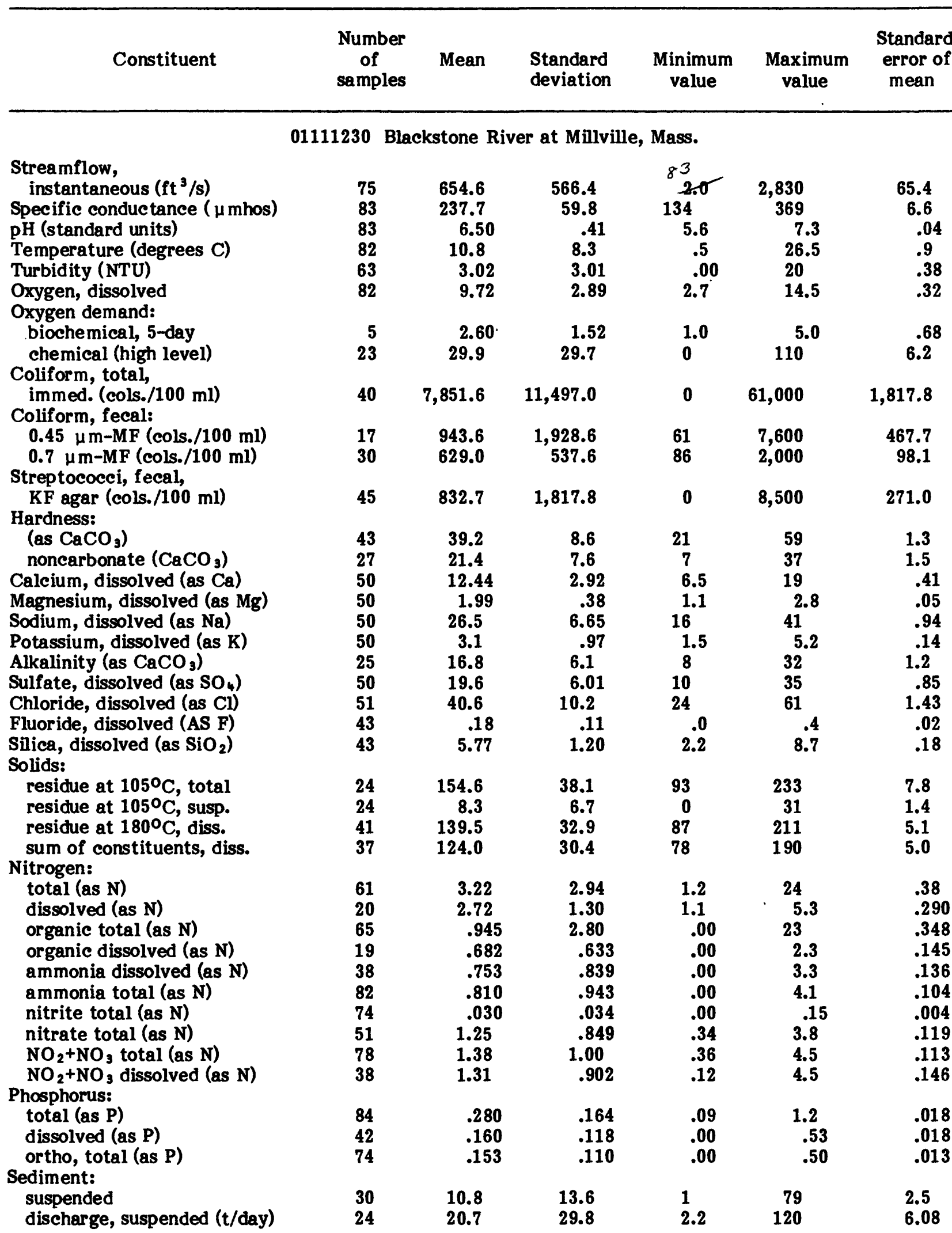


Table 3.-Summary of measure ments of common constituents, nutrients, bacteria, and field measurements for each station (continued)

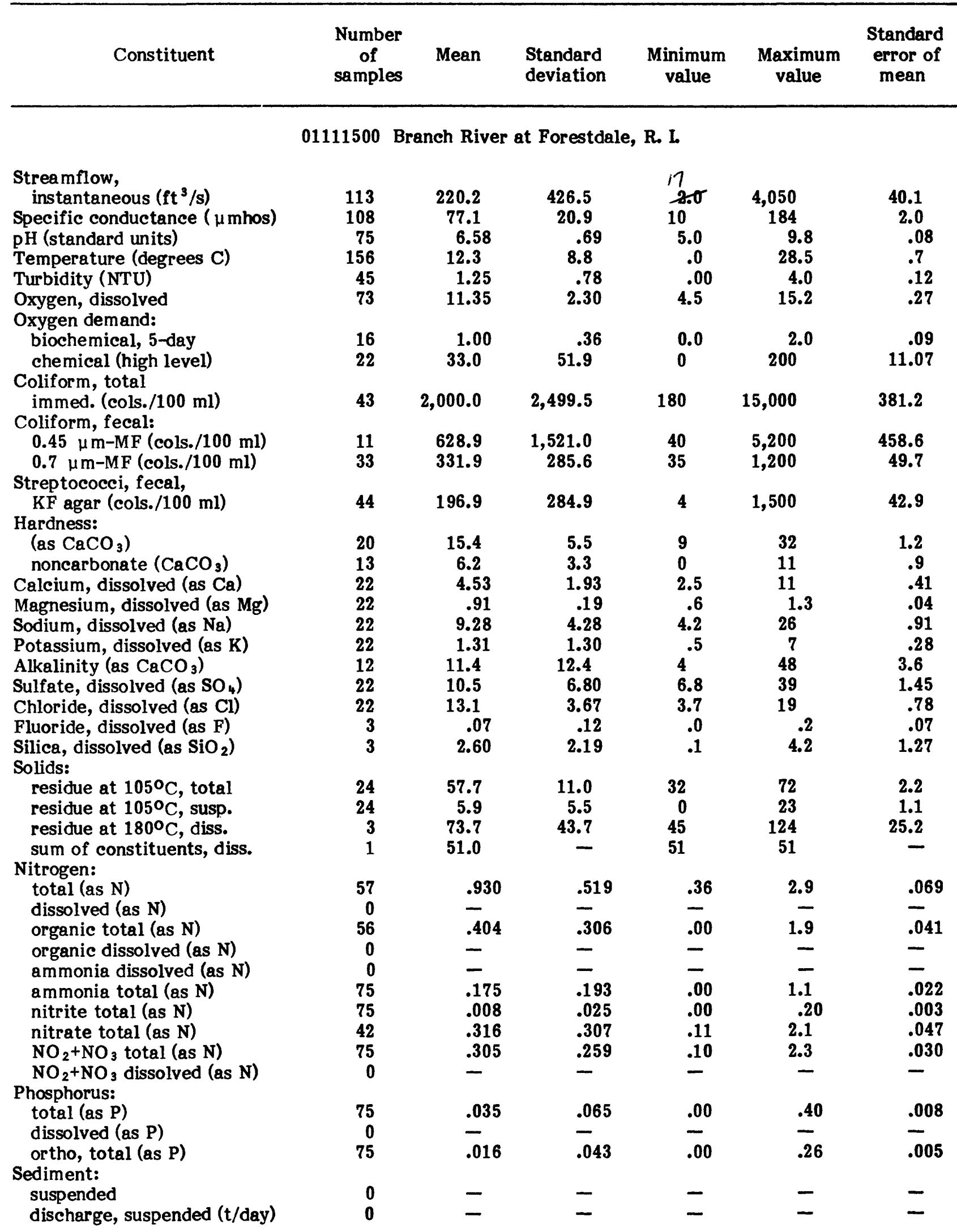


Table 3.-Summary of measurements of common constituents, nutrients, bacteria, and field measurements for each station (continued)

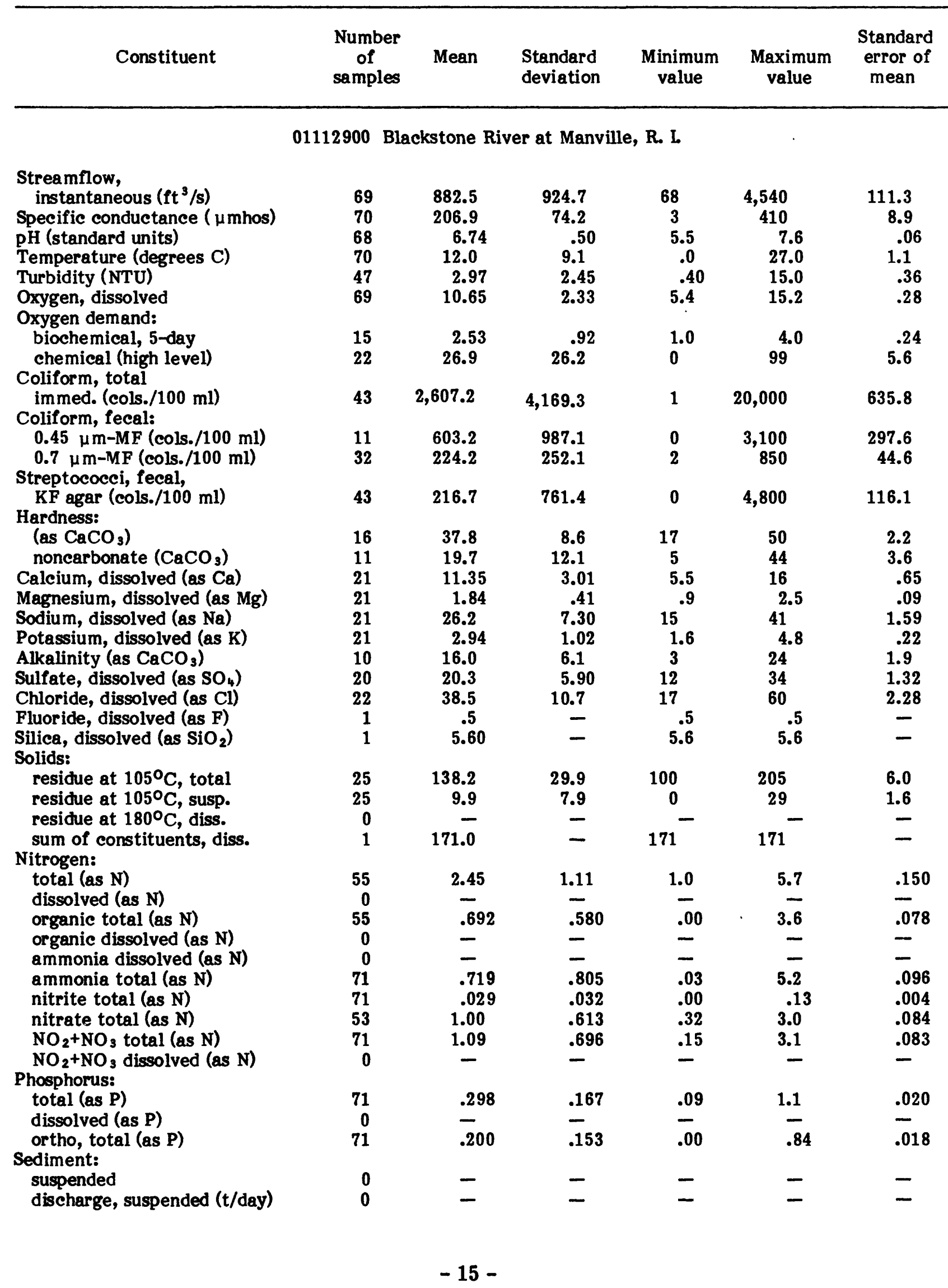


Table 3.-Summary of measurements of common constituents, nutrients, bacteria, and field measurements for each station (continued)

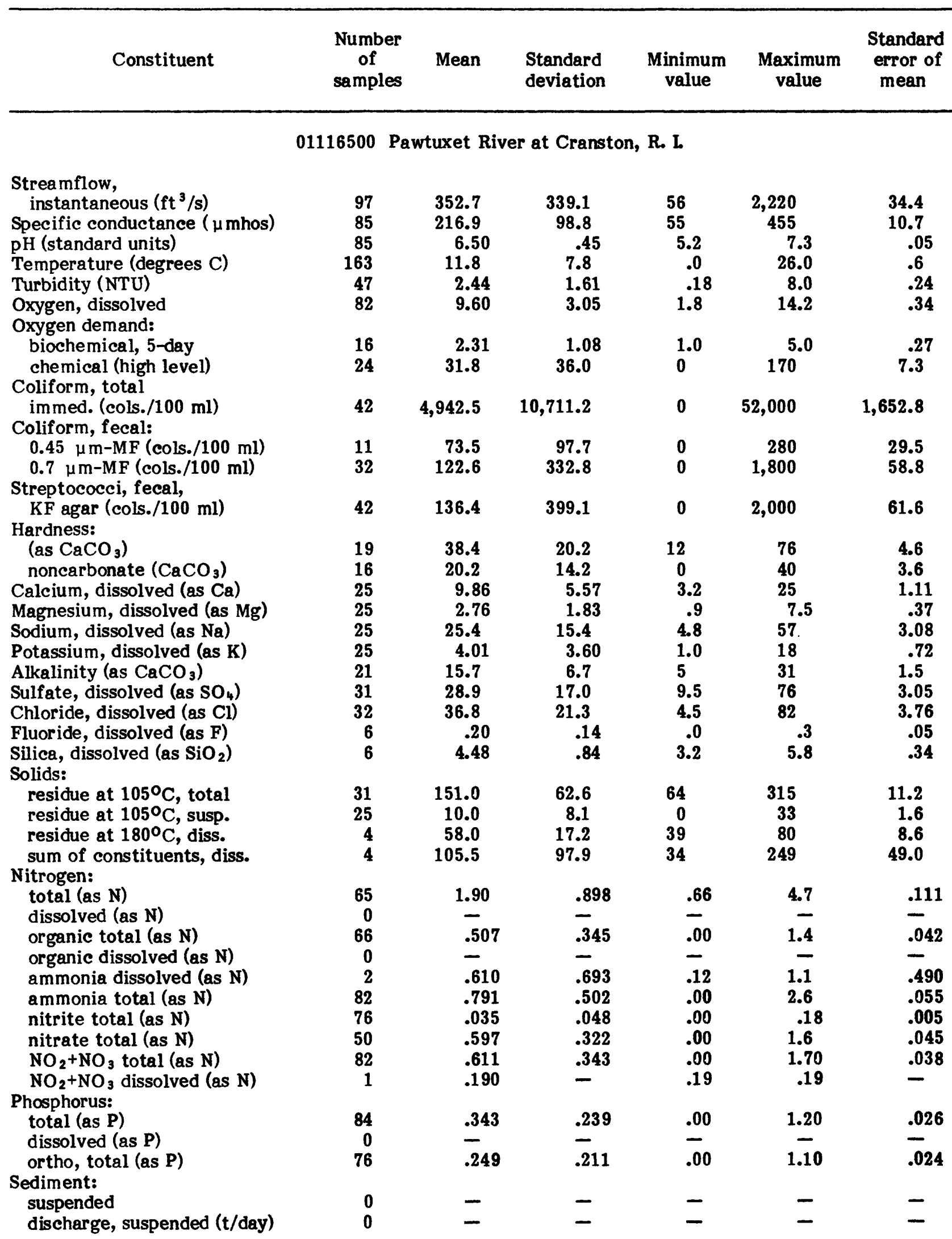


Table 3.-Summary of measurements of common constituents, nutrients, bacteria, and field measurements for each station (continued)

\begin{tabular}{|c|c|c|c|c|c|c|}
\hline \multirow[t]{2}{*}{ Constituent } & $\begin{array}{l}\text { Number } \\
\text { of } \\
\text { samples }\end{array}$ & Mean & $\begin{array}{l}\text { Standard } \\
\text { deviation }\end{array}$ & $\begin{array}{l}\text { Minimum } \\
\text { value }\end{array}$ & $\begin{array}{l}\text { Maximum } \\
\text { value }\end{array}$ & \multirow[t]{2}{*}{$\begin{array}{l}\text { Standard } \\
\text { error of } \\
\text { mean }\end{array}$} \\
\hline & 01116617 & Pawtuxet Riv & rer at Pawtuxe & R. I. & . & \\
\hline \multicolumn{7}{|l|}{ Streamflow, } \\
\hline Specific conductance ( $\mu$ mhos) & 72 & $\begin{array}{l}479.4 \\
254.1\end{array}$ & $\begin{array}{l}497.7 \\
108.6\end{array}$ & $\begin{array}{r}136 \\
10\end{array}$ & $\begin{array}{r}3,370 \\
560\end{array}$ & $\begin{array}{l}59.1 \\
12.8\end{array}$ \\
\hline pH (standard units) & 72 & 6.57 & .41 & 5.7 & 7.5 & .05 \\
\hline Temperature (degrees C) & 74 & 11.3 & 8.2 & .0 & 25.0 & 1.0 \\
\hline Turbidity (NTU) & 47 & 2.67 & 2.29 & .40 & 15.0 & .33 \\
\hline Oxygen, dissolved & 73 & 8.75 & 3.59 & .5 & 14.6 & .42 \\
\hline \multicolumn{7}{|l|}{ Oxygen demand: } \\
\hline $\begin{array}{l}\text { bioche mical, 5-day } \\
\text { chemical (high level) }\end{array}$ & $\begin{array}{l}22 \\
23\end{array}$ & $\begin{array}{l}7.77 \\
40.4\end{array}$ & $\begin{array}{l}4.05 \\
28.9\end{array}$ & $\begin{array}{l}3.0 \\
0\end{array}$ & $\begin{array}{l}16 \\
98\end{array}$ & $\begin{array}{l}.86 \\
6.0\end{array}$ \\
\hline \multicolumn{7}{|l|}{ Coliform, total } \\
\hline immed. (cols./100 ml) & 48 & $77,206.1$ & $173,969.8$ & 1, & $, 100,000$ & $25,110.4$ \\
\hline \multicolumn{7}{|l|}{ Coliform, fecal: } \\
\hline $\begin{array}{l}0.45 \mu \mathrm{m}-\mathrm{MF} \text { (cols. } / 100 \mathrm{ml}) \\
0.7 \mu \mathrm{m}-\mathrm{MF}(\text { cols. } / 100 \mathrm{ml})\end{array}$ & $\begin{array}{l}11 \\
40\end{array}$ & $\begin{array}{l}139.7 \\
337.5\end{array}$ & $\begin{array}{l}233.6 \\
424.2\end{array}$ & $\begin{array}{l}4 \\
0\end{array}$ & $\begin{array}{r}800 \\
2,000\end{array}$ & $\begin{array}{l}70.4 \\
67.1\end{array}$ \\
\hline \multicolumn{7}{|l|}{ Streptococei, fecal, } \\
\hline $\mathrm{KF}$ agar (cols. $/ 100 \mathrm{ml}$ ) & 47 & 341.0 & 674.3 & 0 & 4,000 & 98.4 \\
\hline \multicolumn{7}{|l|}{ Hardness: } \\
\hline$\left(\right.$ as $\left.\mathrm{CaCO}_{3}\right)$ & 14 & 46.0 & 13.8 & 19 & 64 & 3.7 \\
\hline noncarbonate $\left(\mathrm{CaCO}_{3}\right)$ & 10 & 23.4 & 12.6 & 5 & 47 & 4.0 \\
\hline Calcium, dissolved (as Ca) & 19 & 11.39 & 4.13 & 5.5 & 19 & .95 \\
\hline Magnesium, dissolved (as $\mathrm{Mg}$ ) & 19 & 2.93 & 1.28 & 1.2 & 5.4 & .30 \\
\hline Sodium, dissolved (as Na) & 19 & 33.2 & 16.8 & 13 & 69 & 3.86 \\
\hline Potassium, dissolved (as $\mathrm{K}$ ) & 19 & 4.09 & 2.20 & 1.6 & 10 & .50 \\
\hline Alkalinity $\left(\mathrm{aS} \mathrm{CaCO}_{3}\right)$ & 9 & 20.4 & 4.6 & 14 & 26 & 1.5 \\
\hline Sulfate, dissolved (as $\mathrm{SO}_{4}$ ) & 19 & 35.8 & 19.8 & 14 & 85 & 4.5 \\
\hline Chloride, dissolved (as $\mathrm{Cl}$ ) & 20 & 40.4 & 17.0 & 20 & 75 & 3.81 \\
\hline Fluoride, dissolved (as F) & $\mathbf{0}$ & - & - & - & - & - \\
\hline Silica, dissolved (as $\mathrm{SiO}_{2}$ ) & $\mathbf{0}$ & - & - & - & - & - \\
\hline \multicolumn{7}{|l|}{ Solids: } \\
\hline residue at $105^{\circ} \mathrm{C}$, total & 25 & 165.6 & 64.2 & 75 & 325 & 12.8 \\
\hline residue at $105^{\circ} \mathrm{C}$, susp. & 25 & 11.8 & 7.7 & 4 & 32 & 1.5 \\
\hline residue at $180^{\circ} \mathrm{C}$, diss. & $\mathbf{0}$ & - & - & - & - & - \\
\hline sum of constituents, diss. & 0 & - & - & - & - & - \\
\hline \multicolumn{7}{|l|}{ Nitrogen: } \\
\hline total (as N) & 58 & 2.74 & 1.43 & .51 & 6.6 & .187 \\
\hline dissolved (as N) & $\mathbf{0}$ & - & - & - & - & - \\
\hline organic total (as $\mathrm{N}$ ) & 58 & .707 & .505 & .00 & 2.3 & .066 \\
\hline organic dissolved (as N) & $\mathbf{0}$ & - & - & - & - & - \\
\hline ammonia dissolved (as $\mathrm{N}$ ) & $\mathbf{0}$ & - & - & - & - & - \\
\hline a mmonia total (as N) & 77 & 1.37 & 1.07 & .00 & 4.8 & .122 \\
\hline nitrite total (as N) & 77 & .049 & .055 & .00 & .26 & .006 \\
\hline nitrate total (as N) & 56 & .621 & .262 & .07 & 1.4 & .035 \\
\hline $\mathrm{NO}_{2}+\mathrm{NO}_{3}$ total (as $\left.\mathrm{N}\right)$ & 77 & .683 & .300 & .11 & 1.6 & .034 \\
\hline $\mathrm{NO}_{2}+\mathrm{NO}_{3}$ dissolved (as $\mathrm{N}$ ) & 0 & - & - & - & - & - \\
\hline \multicolumn{7}{|l|}{ Phosphorus: } \\
\hline total (as P) & 77 & .501 & .355 & .10 & 2.0 & .040 \\
\hline dissolved (as P) & $\mathbf{0}$ & - & - & - & - & - \\
\hline ortho, total (as P) & 77 & .405 & .332 & .00 & 1.4 & .038 \\
\hline \multicolumn{7}{|l|}{ Sediment: } \\
\hline suspended & 0 & - & - & - & - & - \\
\hline discharge, suspended ( $t /$ day) & 0 & - & - & - & - & - \\
\hline
\end{tabular}


Table 3.-Summary of measurements of common constituents, nutrients, bacteria, and field measurements for each station (continued)

\begin{tabular}{|c|c|c|c|c|c|c|}
\hline Constituent & $\begin{array}{l}\text { Number } \\
\text { of } \\
\text { samples }\end{array}$ & Mean & $\begin{array}{l}\text { Standard } \\
\text { deviation }\end{array}$ & $\begin{array}{l}\text { Minimum } \\
\text { value }\end{array}$ & $\begin{array}{l}\text { Maximum } \\
\text { value }\end{array}$ & $\begin{array}{l}\text { Standard } \\
\text { error of } \\
\text { mean }\end{array}$ \\
\hline & \multicolumn{5}{|c|}{01118500 Pawcatuck River at Westerly, R. I. } & \\
\hline \multicolumn{7}{|l|}{$\begin{array}{l}\text { Streamflow, } \\
\text { instantaneus }\left(\mathrm{ft}^{3} / \mathrm{s}\right)\end{array}$} \\
\hline Specific conductance ( $\mu \mathrm{mhos})$ & 141 & 100.1 & 33.1 & 52 & 222 & 2.8 \\
\hline $\mathrm{pH}$ (standard units) & 112 & 6.55 & .53 & 5.4 & 8.9 & .05 \\
\hline Temperature (degrees C) & 153 & 11.4 & 8.4 & .0 & 30.0 & .7 \\
\hline Turbidity (NTU) & 89 & 1.34 & .72 & .50 & 6.0 & .08 \\
\hline Oxygen, dissolved & 108 & 11.11 & 2.05 & 6.9 & 15.2 & .20 \\
\hline \multicolumn{7}{|l|}{ Oxygen demand: } \\
\hline $\begin{array}{l}\text { biochemical, 5-day } \\
\text { chemical (high level) }\end{array}$ & $\begin{array}{l}25 \\
23\end{array}$ & $\begin{array}{l}1.00 \\
20.0\end{array}$ & 13.91 & $\begin{array}{l}0.0 \\
0\end{array}$ & $\begin{array}{ll}2.0 \\
58\end{array}$ & 2.08 \\
\hline \multicolumn{7}{|l|}{ Coliform, total } \\
\hline immed. (cols. $/ 100 \mathrm{ml}$ ) & 57 & $4,981.9$ & $6,198.9$ & 78 & 23,000 & 821.0 \\
\hline \multicolumn{7}{|l|}{ Coliform, fecal: } \\
\hline $\begin{array}{l}0.45 \mu \mathrm{m}-\mathrm{MF} \text { (cols. } / 100 \mathrm{ml}) \\
0.7 \mu \mathrm{m}-\mathrm{MF}(\text { cols. } / 100 \mathrm{ml})\end{array}$ & $\begin{array}{l}60 \\
41\end{array}$ & $\begin{array}{l}168.9 \\
203.4\end{array}$ & $\begin{array}{l}306.7 \\
324.3\end{array}$ & $\begin{array}{l}6 \\
1\end{array}$ & $\begin{array}{l}2,000 \\
2,000\end{array}$ & $\begin{array}{l}39.6 \\
50.6\end{array}$ \\
\hline \multicolumn{7}{|l|}{ Streptococci, fecal, } \\
\hline $\mathrm{KF}$ agar (cols. $/ 100 \mathrm{ml}$ ) & 98 & 840.2 & $2,557.7$ & 2 & 22,000 & 258.4 \\
\hline \multicolumn{7}{|l|}{ Hardness: } \\
\hline$\left(\mathrm{as} \mathrm{CaCO}_{3}\right)$ & 68 & 15.8 & 3.8 & 10 & 26 & .5 \\
\hline noncarbonate $\left(\mathrm{CaCO}_{3}\right)$ & 47 & 4.6 & 2.9 & $\mathbf{0}$ & 11 & .4 \\
\hline Calcium, dissolved (as Ca) & 74 & 4.15 & 1.00 & 2.5 & 6.8 & .12 \\
\hline Magnesium, dissolved (as Mg) & 74 & 1.30 & .39 & .7 & 3.0 & .05 \\
\hline Sodium, dissolved (as Na) & 66 & 12.11 & 6.10 & 5.1 & 34 & .75 \\
\hline Potassium, dissolved (as $\mathrm{K}$ ) & 66 & 1.09 & .30 & .4 & 1.9 & .04 \\
\hline Alkalinity (as $\mathrm{CaCO}_{3}$ ) & 46 & 12.8 & 10.1 & 0 & 58 & 1.5 \\
\hline Sulfate, dissolved (as $\mathrm{SO}_{4}$ ) & 91 & 14.4 & 6.64 & 6.8 & 40 & .70 \\
\hline Chloride, dissolved (as $\mathrm{Cl}$ ) & 101 & 12.3 & 3.41 & 5.2 & 34 & .34 \\
\hline Fluoride, dissolved (as F) & 55 & .12 & .07 & .0 & .3 & .01 \\
\hline Silica, dissolved (as $\mathrm{SiO}_{2}$ ) & 63 & 6.76 & 2.03 & 2.7 & 11 & .26 \\
\hline \multicolumn{7}{|l|}{ Solids: } \\
\hline residue at $105^{\circ} \mathrm{C}$, total & 96 & 78.4 & 22.5 & 44 & 172 & 2.3 \\
\hline residue at $105^{\circ} \mathrm{C}$, susp. & 19 & 8.0 & 12.6 & 0 & 58 & 2.9 \\
\hline residue at $180^{\circ} \mathrm{C}$, diss. & 94 & 73.9 & 20.9 & 43 & 164 & 2.2 \\
\hline sum of constituents, diss. & 50 & 58.7 & 19.4 & 28 & 125 & 2.7 \\
\hline \multicolumn{7}{|l|}{ Nitrogen: } \\
\hline total (as N) & 93 & .884 & .490 & .18 & 4.6 & .051 \\
\hline dissolved (as $\mathrm{N}$ ) & 19 & .875 & .319 & .45 & 1.4 & .073 \\
\hline organic total (as N) & 93 & .427 & .218 & .01 & 1.2 & .023 \\
\hline organic dissolved (as $\mathrm{N}$ ) & 26 & .376 & .163 & .07 & .77 & .032 \\
\hline ammonia dissolved (as $N$ ) & 56 & .072 & .240 & .00 & 1.8 & .032 \\
\hline a mmonia total (as $N$ ) & 84 & .041 & .038 & .00 & .19 & .004 \\
\hline nitrite total (as $N$ ) & 72 & .004 & .006 & .00 & .02 & .001 \\
\hline nitrate total (as N) & 34 & .405 & .217 & .00 & .84 & .037 \\
\hline $\mathrm{NO}_{2}+\mathrm{NO}_{3}$ total (as $\left.\mathrm{N}\right)$ & 107 & .387 & .222 & .10 & 1.6 & .022 \\
\hline $\mathrm{NO}_{2}+\mathrm{NO}_{3}$ dissolved (as $\mathrm{N}$ ) & 33 & .412 & .217 & .00 & .90 & .038 \\
\hline \multicolumn{7}{|l|}{ Phosphorus: } \\
\hline total (as P) & 107 & .048 & .091 & .00 & .94 & .009 \\
\hline dissolved (as P) & 80 & .030 & .024 & .00 & .10 & .003 \\
\hline ortho, total (as P) & 64 & .019 & .020 & .00 & .09 & .003 \\
\hline \multicolumn{7}{|l|}{ Sediment: } \\
\hline suspended & 48 & 13.5 & 29.7 & 1 & 206 & 4.3 \\
\hline discharge, suspended ( $t /$ day) & 45 & 25.3 & 59.1 & .66 & 389 & 8.8 \\
\hline
\end{tabular}


Trace elements, also frequently called minor elements, are those that commonly occur in relatively smaller amounts in natural water. Many are of concern because, even in trace quantities, they may be toxic to people, to aquatic plants and animals, or to crops when present in irrigation water. Table 4 summarizes trace element data for the six stations. Table 5 lists selected criteria and the maximum measured concentration of 12 trace elements which are measured at the six sites. Unless otherwise stated, the criteria in the following discussion pertain to drinking water (U.S. Environmental Protection Agency, 1976).

The most stringent standard for arsenic is a maximum of $50 \mu \mathrm{g} / \mathrm{L}$ for domestic water supplies. Of the Rhode Island water quality sites, the highest measured concentration for arsenic was $6 \mu \mathrm{g} / \mathrm{L}$ at the Blackstone River at Millville, Mass.

The maximum concentration of barium was $100 \mu \mathrm{g} / \mathrm{L}$ at the Blackstone River at Millville, Mass., and at the Pawcatuck River at Westerly. This is less than the drinking water standard of $1,000 \mu \mathrm{g} / \mathrm{L}$.

The suggested criteria for boron is a maximum of $750 \mu \mathrm{g} / \mathrm{L}$. for long-term irrigation on sensitive crops. While irrigation is not a major use of the water from the six sites, boron levels were less than the maximum acceptable limit for irrigation. The maximum concentration measured was $270 \mu \mathrm{g} / \mathrm{L}$ at the Blackstone River at Millville, Mass.

The drinking water standard for cadmium is $10 \mu \mathrm{g} / \mathrm{L}$. This value was reached in samples from both Blackstone River at Millville, Mass., and Pawcatuck River at Westerly in the total recoverable phase. The maximum dissolved value was $8 \mu \mathrm{g} / \mathrm{L}$ at Blackstone River at Millville, Mass., and $3 \mu \mathrm{g} / \mathrm{L}$ at Pawcatuck River at Westerly. These values exceed the criterion for aquatic life protection of sensitive fish species of $0.4 \mu \mathrm{g} / \mathrm{L}$ of cadmium in soft water. The criterion for other less sensitive aquatic species is $4.0 \mu \mathrm{g} / \mathrm{L}$ in soft water.

Chromium has a drinking water standard of $50 \mu \mathrm{g} / \mathrm{L}$ but a less stringent recommended criteria for the protection of aquatic life of $100 \mu \mathrm{g} / \mathrm{L}$. Maximum total recoverable chromium measured at any of the six sites was $50 \mu \mathrm{g} / \mathrm{L}$ at Blackstone River at Manville. All other total recoverable measurements were less than $50 \mu \mathrm{g} / \mathrm{L}$ as were the dissolved values.

Drinking water criteria were set for iron and manganese not because of their toxic effects of the metals but rather to eliminate the discoloration of porcelain plumbing fixtures and staining of laundry. Limits of $300 \mu \mathrm{g} / \mathrm{L}$ for iron and $50 \mu \mathrm{g} / \mathrm{L}$ for manganese were established to prevent these problems. A criterion of $1,000 \mu \mathrm{g} / \mathrm{L}$ for iron for the protection of aquatic life has been suggested. Several sites had iron concentrations that exceeded the drinking water standard and the largest total recoverable concentration, 1,900 $\mu \mathrm{g} / \mathrm{L}$ at Blackstone River at Millville, Mass., and the largest dissolved concentration, 1,200 $\mu \mathrm{g} / \mathrm{L}$ at Pawcatuck River at Westerly, exceeded the value set for the protection of aquatic life. Pawcatuck River at Westerly was the only station at which the mean concentrations of both total recoverable and dissolved manganese were below the drinking water criterion.

The criterion for lead in drinking water is $50 \mu \mathrm{g} / \mathrm{L}$. The highest measured total recoverable concentration of lead was $51 \mu \mathrm{g} / \mathrm{L}$ at Blackstone River at Millville, Mass. The highest dissolved concentration was $14 \mu \mathrm{g} / \mathrm{L}$ also at Blackstone River at Millville, Mass.

Mercury has the lowest standard set for drinking water of any of the metals, $2 \mu \mathrm{g} / \mathrm{L}$. Blackstone River at Manville had a total recoverable concentration of $1.8 \mu \mathrm{g} / \mathrm{L}$ which was the highest concentration measured at any of the six sites.

Selenium and silver have drinking water standards of $10 \mu \mathrm{g} / \mathrm{L}$ and $50 \mu \mathrm{g} / \mathrm{L}$, respectively. Measured concentrations of these two constituents at the six sites were less than the standards. The largest selenium concentration was $2 \mu \mathrm{g} / \mathrm{L}$ at Branch River at Forestdale, and the largest silver concentration was $7 \mu \mathrm{g} / \mathrm{L}$ at Pawtuxet River at Pawtuxet.

Standards for zine range from a high of $5,000 \mu \mathrm{g} / \mathrm{L}$ for drinking water down to a proposed level of $30-70 \mu \mathrm{g} / \mathrm{L}$ for the protection of the most sensitive aquatic life (Wentz, 1974, p. 27). The maximum zinc concentration was $470 \mu \mathrm{g} / \mathrm{L}$ at Branch River at Forestdale. However, mean concentrations were generally less than $100 \mu \mathrm{g} / \mathrm{L}$. 
Table 4.-Summary of measurements of trace elements for each station

(Analyses in micrograms per liter except as indicated.)

\begin{tabular}{ccccc}
\hline Constituent & $\begin{array}{c}\text { Number } \\
\text { of } \\
\text { samples }\end{array}$ & Mean & $\begin{array}{c}\text { Standard } \\
\text { deviation }\end{array}$ & $\begin{array}{c}\text { Minimum value } \\
\text { value }\end{array}$ \\
\hline
\end{tabular}

01111230 Blackstone River at Millville, Mass.

\begin{tabular}{|c|c|c|c|c|c|c|}
\hline $\begin{array}{l}\text { Aluminum, total } \\
\text { recoverable (as } \mathrm{Al} \text { ) }\end{array}$ & 1 & 90.0 & - & 90 & 90 & - \\
\hline Aluminum, dissolved (as $\mathrm{Al}$ ) & 6 & 40.0 & 29.0 & 0 & 70 & 11.8 \\
\hline Arsenic, dissolved (as As) & 20 & 2.4 & 1.3 & $\mathbf{0}$ & 6 & .3 \\
\hline Arsenic, total (as As) & 17 & 2.8 & 1.6 & 1 & 6 & .4 \\
\hline Barium, dissolved (as Ba) & 20 & 35.1 & 25.6 & $\mathbf{0}$ & 100 & 5.7 \\
\hline Barium, total recoverable (as Ba) & 16 & 37.5 & 50.0 & $\mathbf{0}$ & 100 & 12.5 \\
\hline Beryllium, dissolved (Be) & 6 & .1 & .2 & 0 & 5 & .1 \\
\hline Beryllium, & & & & & & \\
\hline total recoverable (as $\mathrm{Be}$ ) & 2 & .0 & .0 & $\mathbf{0}$ & 0 & .0 \\
\hline Boron, total recoverable (as B) & 4 & 155 & 87.4 & 60 & 270 & 43.7 \\
\hline Cadmium, dissolved (as Cd) & 20 & 2.5 & 2.3 & 0 & 8 & .5 \\
\hline Cadmium, total & & & & & & \\
\hline recoverable (as Cd) & 15 & 3.8 & 2.6 & 1 & 10 & .7 \\
\hline Chromium, dissolved (as $\mathrm{Cr}$ ) & 20 & 6.3 & 9.2 & $\mathbf{0}$ & 30 & 2.1 \\
\hline Chromium, total & & & & & & \\
\hline recoverable (as $\mathrm{Cr}$ ) & 17 & 18.2 & 11.9 & $\mathbf{0}$ & 40 & 2.9 \\
\hline Cobalt, dissolved (as Co) & 20 & .5 & 1.0 & $\mathbf{0}$ & 3 & .2 \\
\hline Cobalt, total recoverable (Co) & 16 & .9 & 1.2 & $\mathbf{0}$ & 4 & .3 \\
\hline Copper, dissolved (as Cu) & 20 & 12.1 & 5.9 & 6 & 31 & 1.3 \\
\hline Copper, total recoverable (as $\mathrm{Cu}$ ) & 16 & 24.8 & 7.6 & 15 & 39 & 1.9 \\
\hline Iron, total recoverable (as $\mathrm{Fe}$ ) & 17 & 903 & 412 & 490 & 1,900 & 100.0 \\
\hline Iron, dissolved (as $\mathrm{Fe}$ ) & 21 & 223 & 106 & 58 & 420 & 23.2 \\
\hline Lead, dissolved (as Pb) & 20 & 3.4 & $\mathbf{3 . 0}$ & $\mathbf{0}$ & 14 & .7 \\
\hline Lead, total recoverable (as $\mathrm{Pb}$ ) & 17 & 16.9 & 11.7 & 4 & 51 & 2.8 \\
\hline Lithium, dissolved (as $\mathrm{Li}$ ) & 6 & 8.2 & 10.9 & $\mathbf{0}$ & 30 & 4.4 \\
\hline Lithium, total recoverable (as $\mathrm{Li}$ ) & 2 & .0 & .0 & 0 & 0 & .0 \\
\hline Manganese, total & & & & & & \\
\hline recoverable (as $\mathrm{Mn}$ ) & 18 & 113 & 49.9 & 30 & 200 & 11.8 \\
\hline Manganese, dissolved (as Mn) & 21 & 95.4 & $\mathbf{5 4 . 0}$ & 15 & 230 & 11.8 \\
\hline Mercury, dissolved (as Hg) & 20 & .1 & .1 & 0 & 2 & .0 \\
\hline Mercury, total & & & & & & \\
\hline recoverable (as $\mathrm{Hg}$ ) & 17 & .0 & .0 & $\mathbf{0}$ & 1 & .0 \\
\hline Molybdenum, dissolved (as Mo) & 6 & 1.7 & 4.1 & $\mathbf{0}$ & 10 & 1.7 \\
\hline Molybdenum, total & & & & & & \\
\hline recoverable (as Mo) & 1 & .0 & - & 0 & $\mathbf{0}$ & - \\
\hline Nickel, dissolved (as Ni) & 18 & 35.1 & 28.6 & 9 & 130 & 6.7 \\
\hline Nickel, total recoverable (as Ni) & 16 & 39.7 & 33.1 & 0 & 130 & 8.3 \\
\hline Selenium, dissolved (as Se) & 20 & .1 & .3 & $\mathbf{0}$ & 1 & .1 \\
\hline Selenium, total (as Se) & 17 & .1 & .3 & 0 & 1 & .1 \\
\hline Silver, dissolved (as Ag) & 19 & .2 & .5 & $\mathbf{0}$ & 2 & .1 \\
\hline Silver, total recoverable (as Ag) & 20 & .1 & .3 & $\mathbf{0}$ & 1 & .1 \\
\hline Strontium, dissolved (as Sr) & 6 & 66.8 & 11.1 & 56 & 86 & 4.5 \\
\hline Strontium, total & & & & & & \\
\hline recoverable (as Sr) & 2 & 90.0 & 14.1 & 80 & 100 & 10.0 \\
\hline Vanadium, dissolved (as V) & 6 & 1.0 & 2.4 & $\mathbf{0}$ & 6 & 1.0 \\
\hline Zinc, dissolved (as Zn) & 20 & 76.5 & 48.9 & 30 & 220 & 10.9 \\
\hline Zinc, total recoverable (as $\mathrm{Zn}$ ) & 17 & 98.2 & 46.9 & 40 & 200 & 11.4 \\
\hline
\end{tabular}


Table 4.-Summary of measurements of trace elements for each station (continued)

\begin{tabular}{ccccc}
\hline Constituent & $\begin{array}{c}\text { Number } \\
\text { of } \\
\text { samples }\end{array}$ & Mean & $\begin{array}{c}\text { Standard } \\
\text { deviation }\end{array}$ & $\begin{array}{c}\text { Minimum value } \\
\text { Maximum } \\
\text { value }\end{array}$ standard \\
error of \\
mean
\end{tabular}

01111500 Branch River at Forestdale, R. I.

\begin{tabular}{|c|c|c|c|c|c|c|}
\hline $\begin{array}{l}\text { Aluminum, total } \\
\text { recoverable (as Al) }\end{array}$ & 1 & 90.0 & - & 90 & 90 & 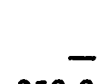 \\
\hline Aluminum, dissolved (as $\mathrm{Al}$ ) & 2 & 250 & 354 & 0 & 500 & 250.0 \\
\hline Arsenic, dissolved (as As) & $\mathbf{0}$ & - & - & - & - & - \\
\hline Arsenic, total (as As) & $\mathbf{9}$ & .6 & .5 & $\mathbf{0}$ & 1 & .2 \\
\hline Barium, dissolved (as Ba) & $\mathbf{0}$ & - & - & - & - & - \\
\hline Barium, total recoverable (as $\mathrm{Ba}$ ) & 2 & .0 & .0 & 0 & 0 & .0 \\
\hline Beryllium, dissolved (Be) & $\mathbf{0}$ & - & - & - & - & - \\
\hline $\begin{array}{l}\text { Beryllium, total } \\
\text { recoverable (as Be) }\end{array}$ & & & & & & \\
\hline $\begin{array}{l}\text { recoverable (as Be) } \\
\text { Boron, total recoverable (as B) }\end{array}$ & $\begin{array}{l}2 \\
7\end{array}$ & 2.0 & .0 & $\begin{array}{l}0 \\
0\end{array}$ & $\begin{array}{r}0 \\
50\end{array}$ & $\begin{array}{r}.0 \\
7.5\end{array}$ \\
\hline Cadmium, dissolved (as Cd) & 0 & 25.7 & 19.9 & $\underline{0}$ & SU & 1.0 \\
\hline Cadmium, total & & & & & & \\
\hline recoverable (as Cd) & 7 & .6 & .8 & 0 & 2 & .3 \\
\hline Chromium, dissolved (as $\mathrm{Cr}$ ) & $\mathbf{0}$ & - & - & - & - & - \\
\hline $\begin{array}{l}\text { Chromium, total } \\
\text { recoverable (as Cr) }\end{array}$ & 9 & 100 & 87 & 0 & 20 & 29 \\
\hline Cobalt, dissolved (as Co) & 0 & - & - & - & - & - \\
\hline Cobalt, total recoverable (Co) & 2 & .0 & .0 & $\mathbf{0}$ & 0 & .0 \\
\hline Copper, dissolved (as Cu) & 2 & 70.0 & 56.6 & 30 & 110 & 40.0 \\
\hline Copper, total recoverable (as Cu) & 8 & 13.0 & 9.5 & 3 & 30 & 3.4 \\
\hline Iron, total recoverable (as $\mathrm{Fe}$ ) & 10 & 576 & 301 & 210 & 1000 & 95.3 \\
\hline Iron, dissolved (as Fe) & 2 & 345 & 403 & 60 & 630 & 285.0 \\
\hline Lead, dissolved (as $\mathrm{Pb}$ ) & $\mathbf{0}$ & - & - & - & - & - \\
\hline Lead, total recoverable (as $\mathrm{Pb}$ ) & $\mathbf{9}$ & 8.2 & 9.7 & 1 & 31 & 3.2 \\
\hline Lithium, dissolved (as Li) & 2 & 300 & 424 & 0 & 600 & 300.0 \\
\hline Lithium, total recoverable (as $\mathrm{Li}$ ) & 2 & .0 & .0 & $\mathbf{0}$ & 0 & .0 \\
\hline Manganese, total & & & & & & \\
\hline recoverable (as Mn) & 10 & 120 & 49.0 & 70 & 240 & 15.5 \\
\hline Manganese, dissolved (as Mn) & 2 & .0 & .0 & 0 & $\mathbf{0}$ & .0 \\
\hline Mercury, dissolved (as Hg) & 0 & - & - & - & - & - \\
\hline $\begin{array}{l}\text { Mercury, total } \\
\text { recoverable (as Hg) }\end{array}$ & $\mathbf{9}$ & .0 & .1 & 0 & .1 & .0 \\
\hline Molybdenum, dissolved (as Mo) & 0 & - & - & - & - & - \\
\hline Molybdenum, total & & & & & & \\
\hline recoverable (as Mo) & 1 & 1.0 & - & 1 & 1 & - \\
\hline Nickel, dissolved (as Ni) & 0 & - & $=$ & - & - & - \\
\hline Nickel, total recoverable (as $\mathrm{Ni}$ ) & $\mathbf{9}$ & 7.6 & 7.7 & 2 & 26 & 2.6 \\
\hline Selenium, dissolved (as Se) & $\mathbf{0}$ & - & - & - & - & - \\
\hline Selenium, total (as Se) & $\mathbf{9}$ & .2 & .7 & $\mathbf{0}$ & 2 & .2 \\
\hline Silver, dissolved (as Ag) & 0 & - & - & - & - & - \\
\hline Silver, total recoverable (as Ag) & 7 & .0 & .0 & $\mathbf{0}$ & $\mathbf{0}$ & .0 \\
\hline Strontium, dissolved (as Sr) & 0 & - & - & - & - & - \\
\hline $\begin{array}{l}\text { Strontium, total } \\
\text { recoverable (as Sr) }\end{array}$ & 2 & 75.0 & 21.2 & 60 & 90 & 15.0 \\
\hline Vanadium, dissolved (as V) & 0 & - & - & - & - & - \\
\hline Zinc, dissolved (as Zn) & 2 & 235 & 332 & $\mathbf{0}$ & 470 & 235.0 \\
\hline Zinc, total recoverable (as $\mathrm{Zn}$ ) & 9 & 53.3 & 33.9 & 10 & 120 & 11.3 \\
\hline
\end{tabular}


Table 4.-Summary of measurements of trace elements for each station (continued)

\begin{tabular}{|c|c|c|c|c|c|c|}
\hline Constituent & $\begin{array}{l}\text { Number } \\
\text { of } \\
\text { samples }\end{array}$ & Mean & $\begin{array}{l}\text { Standard } \\
\text { deviation }\end{array}$ & $\begin{array}{l}\text { Minimum } \\
\text { value }\end{array}$ & $\begin{array}{l}\text { Maximum } \\
\text { value }\end{array}$ & $\begin{array}{l}\text { Standard } \\
\text { error of } \\
\text { mean }\end{array}$ \\
\hline
\end{tabular}

01112900 Blackstone River at Manville, R. I.

\begin{tabular}{|c|c|c|c|c|c|c|}
\hline $\begin{array}{l}\text { Aluminum, total } \\
\text { recoverable (as } \mathrm{Al} \text { ) }\end{array}$ & 1 & 50.0 & - & 50 & 50 & - \\
\hline Aluminum, dissolved (as Al) & 0 & - & - & - & - & - \\
\hline Arsenic, dissolved (as As) & $\mathbf{0}$ & - & - & - & - & - \\
\hline Arsenic, total (as As) & 9 & 1.8 & 1.2 & $\mathbf{0}$ & 4 & .4 \\
\hline Barium, dissolved (as Ba) & $\mathbf{0}$ & - & - & - & - & - \\
\hline Barium, total recoverable (as $\mathrm{Ba}$ ) & 2 & .0 & .0 & $\mathbf{0}$ & 0 & .0 \\
\hline Beryllium, dissolved (Be) & $\mathbf{0}$ & - & - & - & - & - \\
\hline Beryllium, total & & & & & & \\
\hline recoverable (as $\mathrm{Be}$ ) & 2 & .0 & .0 & $\mathbf{0}$ & 0 & .0 \\
\hline Boron, total recoverable (as B) & 7 & 136 & 67.5 & 40 & 240 & 25.5 \\
\hline Cadmium, dissolved (as Cd) & $\mathbf{0}$ & - & - & - & - & - \\
\hline Cadmium, total & & & & & & \\
\hline recoverable (as Cd) & 7 & 1.6 & 1.5 & $\mathbf{0}$ & 4 & .6 \\
\hline Chromium, dissolved (as $\mathrm{Cr}$ ) & $\mathbf{0}$ & - & - & - & - & - \\
\hline Chromium, total & & & & & & \\
\hline recoverable (as $\mathrm{Cr}$ ) & 9 & 20.0 & 14.1 & $\mathbf{0}$ & 50 & 4.7 \\
\hline Cobalt, dissolved (as Co) & $\mathbf{0}$ & - & - & - & - & - \\
\hline Cobalt, total recoverable (Co) & 2 & 1.5 & 2.1 & $\mathbf{0}$ & 3 & 1.5 \\
\hline Copper, dissolved (as Cu) & $\mathbf{0}$ & - & - & - & - & - \\
\hline Copper, total recoverable (as $\mathrm{Cu}$ ) & 8 & 23.8 & 12.4 & 12 & 42 & 4.4 \\
\hline Iron, total recoverable (as $\mathrm{Fe}$ ) & 9 & 764 & 318 & 470 & 1500 & 106.2 \\
\hline Iron, dissolved (as $\mathrm{Fe}$ ) & 1 & 320 & - & 320 & 320 & - \\
\hline Lead, dissolved (as Pb) & 0 & - & - & - & - & - \\
\hline Lead, total recoverable (as $\mathrm{Pb}$ ) & 9 & 16.3 & 13.9 & $\mathbf{5}$ & 45 & 4.6 \\
\hline Lithium, dissolved (as $\mathrm{Li}$ ) & 0 & - & - & - & - & - \\
\hline Lithium, total recoverable (as Li) & 2 & .0 & .0 & $\mathbf{0}$ & 0 & .0 \\
\hline Manganese, total & & & & & & \\
\hline recoverable (as Mn) & 9 & 118 & 44.1 & 80 & 220 & 14.7 \\
\hline Manganese, dissolved (as Mn) & 1 & 260 & - & 260 & 260 & - \\
\hline Mercury, dissolved (as Hg) & $\mathbf{0}$ & - & - & - & - & - \\
\hline Mercury, total & & & & & & \\
\hline recoverable (as $\mathrm{Hg}$ ) & 9 & .2 & .6 & $\mathbf{0}$ & 1.8 & .2 \\
\hline Molybdenum, dissolved (as Mo) & 0 & - & - & - & - & - \\
\hline Molybdenum, total & & & & & & \\
\hline recoverable (as Mo) & 1 & 1.0 & - & 1 & 1 & - \\
\hline Nickel, dissolved (as Ni) & 0 & - & - & - & - & - \\
\hline Nickel, total recoverable (as $\mathrm{Ni}$ ) & 8 & 36.1 & 21.1 & 2 & 65 & 7.5 \\
\hline Selenium, dissolved (as Se) & $\mathbf{0}$ & - & - & - & - & - \\
\hline Selenium, total (as Se) & 9 & .1 & .3 & 0 & 1 & .1 \\
\hline Silver, dissolved (as Ag) & $\mathbf{0}$ & - & - & - & - & - \\
\hline Silver, total recoverable (as Ag) & 6 & .2 & .4 & $\mathbf{0}$ & 1 & .2 \\
\hline Strontium, dissolved (as Sr) & $\mathbf{0}$ & - & - & - & - & - \\
\hline Strontium, total & 2 & 90.0 & 14.1 & 80 & 100 & 100 \\
\hline Vanadium, dissolved (as V) & 0 & - & - & - & - & - \\
\hline Zinc, dissolved (as Zn) & 0 & - & - & - & - & - \\
\hline Zine, total recoverable (as $\mathrm{Zn}$ ) & 9 & 78.9 & 40.8 & 30 & 140 & 13.6 \\
\hline
\end{tabular}


Table 4.-Summary of measurements of trace elements for each station (continued)

\begin{tabular}{|c|c|c|c|c|c|c|}
\hline Constituent & $\begin{array}{l}\text { Number } \\
\text { of } \\
\text { samples }\end{array}$ & Mean & $\begin{array}{l}\text { Standard } \\
\text { deviation }\end{array}$ & $\begin{array}{l}\text { Minimum } \\
\text { value }\end{array}$ & $\begin{array}{l}\text { Maximum } \\
\text { value }\end{array}$ & $\begin{array}{l}\text { Standard } \\
\text { error of } \\
\text { mean }\end{array}$ \\
\hline
\end{tabular}

01116500 Pawtuxet River at Cranston, R. I.

\begin{tabular}{|c|c|c|c|c|c|c|}
\hline Aluminum, total & & & & & & \\
\hline $\begin{array}{l}\text { recoverable (as } \mathrm{Al} \text { ) } \\
\text { Aluminum, dissolved (as } \mathrm{Al} \text { ) }\end{array}$ & 2 & 125 & 177 & $\overline{10}$ & $\begin{array}{l}250 \\
100\end{array}$ & $\begin{aligned} 125.0 \\
.0\end{aligned}$ \\
\hline $\begin{array}{l}\text { Aluminum, dissolved (as Al) } \\
\text { Arsenic, dissolved (as As) }\end{array}$ & $\begin{array}{l}2 \\
0\end{array}$ & ${ }^{100}$ & .0 & 100 & ${ }^{100}$ & .0 \\
\hline Arsenic, total (as As) & 12 & .9 & .8 & $\mathbf{0}$ & 3 & .2 \\
\hline Barium, dissolved (as Ba) & $\mathbf{0}$ & - & - & - & - & - \\
\hline Barium, total recoverable (as $\mathrm{Ba}$ ) & 3 & 3.3 & 5.8 & $\boldsymbol{\theta}$ & 10 & 3.3 \\
\hline Beryllium, dissolved (Be) & 0 & - & - & - & - & - \\
\hline Beryllium, total & & & & & & \\
\hline recoverable (as Be) & 3 & .0 & .0 & $\mathbf{0}$ & 0 & .0 \\
\hline Boron, total recoverable (as B) & 7 & 52.9 & 19.8 & 30 & 80 & 7.5 \\
\hline Cadmium, dissolved (as Cd) & 0 & - & - & - & - & - \\
\hline Cadmium, total & & & & & & \\
\hline recoverable (as Cd) & 11 & 1.6 & 1.7 & $\mathbf{0}$ & 4 & .5 \\
\hline Chromium, dissolved (as $\mathrm{Cr}$ ) & 0 & - & - & - & - & - \\
\hline Chromium, total & & & & & & \\
\hline recoverable (as $\mathrm{Cr}$ ) & 15 & 11.7 & 11.6 & 0 & 40 & 3.0 \\
\hline Cobalt, dissolved (as Co) & $\mathbf{0}$ & - & - & - & - & - \\
\hline Cobalt, total recoverable (Co) & 5 & .0 & .0 & 0 & $\mathbf{0}$ & .0 \\
\hline Copper, dissolved (as Cu) & 2 & 35.0 & 21.2 & 20 & 50 & 15.0 \\
\hline Copper, total recoverable (as $\mathrm{Cu}$ ) & 12 & 21.9 & 8.2 & 13 & 41 & 2.4 \\
\hline Iron, total recoverable (as $\mathrm{Fe}$ ) & 16 & 592 & 215 & 180 & 860 & 53.7 \\
\hline Iron, dissolved (as $\mathrm{Fe}$ ) & 3 & 303 & 201 & 80 & 470 & 116.1 \\
\hline Lead, dissolved (as $\mathrm{Pb}$ ) & $\mathbf{0}$ & - & - & - & - & - \\
\hline Lead, total recoverable (as $\mathrm{Pb}$ ) & 13 & 10.1 & 7.0 & 1 & 23 & 1.9 \\
\hline Lithium, dissolved (as Li) & 2 & 300 & .0 & 300 & 300 & .0 \\
\hline Lithium, total recoverable (as $\mathrm{Li}$ ) & 3 & .0 & .0 & 0 & 0 & .0 \\
\hline Manganese, total & & & & & & \\
\hline recoverable (as Mn) & 16 & 179 & 92.0 & 0 & 360 & 23.0 \\
\hline Manganese, dissolved (as Mn) & 3 & 46.7 & 72.3 & $\mathbf{0}$ & 130 & 41.8 \\
\hline Mercury, dissolved (as Hg) & $\mathbf{0}$ & - & - & - & - & - \\
\hline Mercury, total & & & & & & \\
\hline recoverable (as $\mathrm{Hg}$ ) & 12 & .0 & .0 & $\mathbf{0}$ & 1 & .0 \\
\hline Molybdenum, dissolved (as Mo) & $\mathbf{0}$ & - & - & - & - & - \\
\hline Molybdenum, total & & & & & & \\
\hline recoverable (as Mo) & 2 & .0 & .0 & $\mathbf{0}$ & $\mathbf{0}$ & .0 \\
\hline Nickel, dissolved (as Ni) & 0 & - & - & - & - & - \\
\hline Nickel, total recoverable (as $\mathrm{Ni}$ ) & 9 & 19.9 & 19.9 & 2 & 69 & 6.6 \\
\hline Selenium, dissolved (as Se) & $\mathbf{0}$ & - & - & - & - & - \\
\hline Selenium, total (as Se) & 12 & .0 & .0 & $\mathbf{0}$ & 0 & .0 \\
\hline Silver, dissolved (as Ag) & 0 & - & - & - & - & - \\
\hline Silver, total recoverable (as Ag) & 7 & .1 & .4 & $\mathbf{0}$ & 1 & .1 \\
\hline Strontium, dissolved (as Sr) & $\mathbf{0}$ & - & - & - & - & - \\
\hline $\begin{array}{l}\text { Strontium, total } \\
\text { recoverable (as Sr) }\end{array}$ & & & & & & 176 \\
\hline recoverable (as Sr) & 3 & 73.3 & 30.6 & 40 & 100 & 17.6 \\
\hline $\begin{array}{l}\text { Vanadium, dissolved (as V) } \\
\text { Zine, dissolved (as Zn) }\end{array}$ & $\begin{array}{l}0 \\
2\end{array}$ & $15 . \overline{0}$ & 21.2 & $\overline{0}$ & $30^{-}$ & 15.0 \\
\hline Zine, total recoverable (as $\mathrm{Zn}$ ) & 12 & $\mathbf{5 3 . 3}$ & 33.4 & 20 & 130 & 9.6 \\
\hline
\end{tabular}


Table 4.-Summary of measurements of trace elements for each station (continued)

\begin{tabular}{ccccc}
\hline Constituent & $\begin{array}{c}\text { Number } \\
\text { of } \\
\text { samples }\end{array}$ Mean & $\begin{array}{c}\text { Standard } \\
\text { deviation }\end{array}$ & $\begin{array}{c}\text { Minimum value } \\
\text { value }\end{array}$ & $\begin{array}{c}\text { Maximum value } \\
\text { error of } \\
\text { mean }\end{array}$ \\
\hline
\end{tabular}

01116617 Pawtuxet River at Pawtuxet, R. I.

\begin{tabular}{|c|c|c|c|c|c|c|}
\hline $\begin{array}{l}\text { Aluminum, total } \\
\text { recoverable (as Al) }\end{array}$ & 1 & 80.0 & - & 80 & 80 & - \\
\hline Aluminum, dissolved (as Al) & $\mathbf{0}$ & - & - & - & - & - \\
\hline Arsenic, dissolved (as As) & $\mathbf{0}$ & - & - & - & - & - \\
\hline Arsenic, total (as As) & 9 & 1.3 & 1.2 & $\mathbf{0}$ & 4 & .4 \\
\hline Barium, dissolved (as $\mathrm{Ba}$ ) & $\mathbf{0}$ & - & - & - & - & - \\
\hline Barium, total recoverable (as $\mathrm{Ba}$ ) & 2 & .0 & .0 & $\mathbf{0}$ & 0 & .0 \\
\hline Beryllium, dissolved (Be) & $\mathbf{0}$ & - & - & - & - & - \\
\hline \multicolumn{7}{|l|}{ Beryllium, total } \\
\hline recoverable (as $\mathrm{Be}$ ) & 2 & .0 & .0 & $\mathbf{0}$ & $\mathbf{0}$ & .0 \\
\hline Boron, total recoverable (as B) & 7 & 65.7 & 25.1 & 30 & 100 & 9.5 \\
\hline Cadmium, dissolved (as Cd) & $\mathbf{0}$ & - & - & - & - & - \\
\hline \multicolumn{7}{|l|}{ Cadmium, total } \\
\hline recoverable (as Cd) & 7 & 2.4 & 2.1 & $\mathbf{0}$ & 5 & .8 \\
\hline Chromium, dissolved (as $\mathrm{Cr}$ ) & $\mathbf{0}$ & - & - & - & - & - \\
\hline \multicolumn{7}{|l|}{ Chromium, total } \\
\hline recoverable (as $\mathrm{Cr}$ ) & 9 & 12.2 & 8.3 & $\mathbf{0}$ & 20 & 2.8 \\
\hline Cobalt, dissolved (as Co) & 0 & - & $\overline{0}$ & - & - & $\overline{0}$ \\
\hline Cobalt, total recoverable (Co) & 2 & .0 & .0 & $\mathbf{0}$ & 0 & .0 \\
\hline Copper, dissolved (as Cu) & $\mathbf{0}$ & - & - & - & - & - \\
\hline $\begin{array}{l}\text { Copper, total recoverable (as } \mathrm{Cu}) \\
\text { Iron, total recoverable (as } \mathrm{Fe} \text { ) }\end{array}$ & $\begin{array}{l}8 \\
9\end{array}$ & $\begin{array}{l}26.5 \\
777\end{array}$ & $\begin{array}{l}8.2 \\
330\end{array}$ & $\begin{array}{r}16 \\
280\end{array}$ & $\begin{array}{r}38 \\
1400\end{array}$ & $\begin{array}{r}2.9 \\
109.9\end{array}$ \\
\hline Iron, dissolved (as $\mathrm{Fe}$ ) & 0 & - & - & - & - & - \\
\hline Lead, dissolved (as Pb) & $\mathbf{0}$ & - & - & - & - & - \\
\hline Lead, total recoverable (as $\mathrm{Pb}$ ) & 9 & 10.7 & 6.8 & 4 & 25 & 2.3 \\
\hline Lithium, dissolved (as Li) & $\mathbf{0}$ & - & - & - & - & - \\
\hline Lithium, total recoverable (as $\mathrm{Li}$ ) & 2 & .0 & .0 & - & $\mathbf{0}$ & .0 \\
\hline \multicolumn{7}{|l|}{ Manganese, total } \\
\hline Manganese, dissolved (as Mn) & 0 & 226 & 99.3 & 110 & ${ }^{440}$ & 33.1 \\
\hline Mercury, dissolved (as $\mathrm{Hg}$ ) & $\mathbf{0}$ & - & - & - & - & - \\
\hline \multicolumn{7}{|l|}{ Mercury, total } \\
\hline recoverable (as $\mathrm{Hg}$ ) & 9 & .1 & .1 & $\mathbf{0}$ & .2 & .0 \\
\hline Molybdenum, dissolved (as Mo) & $\mathbf{0}$ & - & - & - & - & - \\
\hline \multicolumn{7}{|l|}{ Molybdenum, total } \\
\hline recoverable (as Mo) & 1 & .0 & - & $\mathbf{0}$ & $\mathbf{0}$ & - \\
\hline Nickel, dissolved (as Ni) & $\mathbf{0}$ & - & - & - & - & - \\
\hline Nickel, total recoverable (as $\mathrm{Ni}$ ) & 9 & 23.4 & 15.2 & 3 & 52 & 5.1 \\
\hline Selenium, dissolved (as Se) & $\mathbf{0}$ & - & - & - & - & - \\
\hline Selenium, total (as Se) & 9 & .0 & .0 & $\mathbf{0}$ & 0 & .0 \\
\hline Silver, dissolved (as Ag) & $\mathbf{0}$ & - & - & - & - & - \\
\hline Silver, total recoverable (as $\mathrm{Ag}$ ) & 7 & 1.1 & 2.6 & $\mathbf{0}$ & 7 & 1.0 \\
\hline Strontium, dissolved (as Sr) & $\mathbf{0}$ & - & - & - & - & - \\
\hline \multicolumn{7}{|l|}{ Strontium, total } \\
\hline recoverable (as Sr) & 2 & 85.0 & 21.2 & 70 & 100 & 15.0 \\
\hline Vanadium, dissolved (as V) & $\mathbf{0}$ & - & - & - & - & - \\
\hline Zinc, dissolved (as Zn) & $\mathbf{0}$ & - & - & - & - & - \\
\hline Zinc, total recoverable (as $\mathrm{Zn}$ ) & 9 & 65.6 & 30.0 & 30 & 120 & 10.0 \\
\hline
\end{tabular}


Table 4.-Summary of measurements of trace elements for each station (continued)

\begin{tabular}{|c|c|c|c|c|c|c|}
\hline Constituent & $\begin{array}{l}\text { Number } \\
\text { of } \\
\text { samples }\end{array}$ & Mean & $\begin{array}{l}\text { Standard } \\
\text { deviation }\end{array}$ & $\begin{array}{l}\text { Minimum } \\
\text { value }\end{array}$ & $\begin{array}{l}\text { Maximum } \\
\text { value }\end{array}$ & $\begin{array}{l}\text { Standard } \\
\text { error of } \\
\text { mean }\end{array}$ \\
\hline \multicolumn{7}{|c|}{01118500 Pawcatuck River at Westerly, R. I. } \\
\hline \multicolumn{7}{|l|}{ Aluminum, total } \\
\hline Aluminum, dissolved (as Al) & 8 & 78.8 & 62.2 & 0 & 170 & 22.0 \\
\hline Arsenic, dissolved (as As) & 25 & .5 & .6 & 0 & 2 & .1 \\
\hline Arsenic, total (as As) & 23 & 1.0 & .6 & 0 & 2 & .1 \\
\hline Barium, dissolved (as Ba) & 25 & 17.4 & 17.7 & $\mathbf{0}$ & 79 & 3.5 \\
\hline Barium, total recoverable (as $\mathrm{Ba}$ ) & 20 & 21.0 & 40.8 & 0 & 100 & 9.1 \\
\hline Beryllium, dissolved (Be) & 6 & .0 & .0 & 0 & 0 & .0 \\
\hline \multicolumn{7}{|l|}{ Beryllium, total } \\
\hline recoverable (as Be) & 2 & .0 & .0 & 0 & 0 & .0 \\
\hline Boron, total recoverable (as B) & 5 & 46.0 & 34.4 & $\mathbf{0}$ & 80 & 15.4 \\
\hline Cadmium, dissolved (as Cd) & 49 & .7 & .8 & $\mathbf{0}$ & 3 & .1 \\
\hline Cadmium, total & & & & . & & \\
\hline recoverable (as Cd) & 22 & 1.3 & 2.5 & $\mathbf{0}$ & 10 & .5 \\
\hline Chromium, dissolved (as $\mathrm{Cr}$ ) & 50 & 2.3 & 4.3 & $\mathbf{0}$ & 20 & .6 \\
\hline \multicolumn{7}{|l|}{ Chromium, total } \\
\hline recoverable (as $\mathrm{Cr}$ ) & 24 & 13.3 & 10.1 & $\mathbf{0}$ & 30 & 2.1 \\
\hline Cobalt, dissolved (as Co) & 25 & .6 & 1.0 & $\mathbf{0}$ & 3 & .2 \\
\hline Cobalt, total recoverable (Co) & 21 & .7 & 1.1 & 0 & 3 & .3 \\
\hline Copper, dissolved (as Cu) & 89 & 4.9 & 12.1 & $\mathbf{0}$ & 100 & 1.3 \\
\hline Copper, total recoverable (as $\mathrm{Cu}$ ) & 25 & 8.8 & 12.7 & 1 & 60 & 2.5 \\
\hline Iron, total recoverable (as $\mathrm{Fe}$ ) & 28 & 449 & 221 & 130 & 1,100 & 41.8 \\
\hline Iron, dissolved (as Fe) & 35 & 304 & 196 & 120 & 1,200 & 33.1 \\
\hline Lead, dissolved (as $\mathrm{Pb}$ ) & 48 & 2.1 & 1.9 & 0 & 9 & .3 \\
\hline Lead, total recoverable (as $\mathrm{Pb}$ ) & 22 & 7.3 & 7.1 & $\mathbf{0}$ & 34 & 1.5 \\
\hline Lithium, dissolved (as $\mathrm{Li}$ ) & 8 & 12.5 & 35.4 & $\mathbf{0}$ & 100 & 12.5 \\
\hline Lithium, total recoverable (as $\mathrm{Li}$ ) & 4 & 25.0 & 50.0 & $\mathbf{0}$ & 100 & 25.0 \\
\hline \multicolumn{7}{|l|}{ Manganese, total } \\
\hline recoverable (as $\mathrm{Mn}$ ) & 28 & 41.1 & 25.1 & 0 & 110 & 4.8 \\
\hline Manganese, dissolved (as Mn) & 34 & 37.8 & 21.4 & $\mathbf{0}$ & 100 & 3.7 \\
\hline Mercury, dissolved (as $\mathrm{Hg}$ ) & 25 & .1 & .2 & $\mathbf{0}$ & .7 & .0 \\
\hline \multicolumn{7}{|l|}{ Mercury, total } \\
\hline recoverable (as $\mathrm{Hg}$ ) & 23 & .1 & .1 & $\mathbf{0}$ & .3 & .0 \\
\hline Molybdenum, dissolved (as Mo) & 6 & .0 & .0 & 0 & 0 & .0 \\
\hline \multicolumn{7}{|l|}{ Molybdenum, total } \\
\hline recoverable (as Mo) & 1 & 1.0 & - & 1 & 1 & $\overline{0}$ \\
\hline Nickel, dissolved (as Ni) & 43 & 1.4 & 1.8 & $\mathbf{0}$ & 10 & .3 \\
\hline Nickel, total recoverable (as $\mathrm{Ni}$ ) & 17 & 3.8 & 3.9 & $\mathbf{0}$ & 14 & .9 \\
\hline Selenium, dissolved (as Se) & 25 & .0 & .0 & 0 & $\mathbf{0}$ & .0 \\
\hline Selenium, total (as Se) & 23 & .0 & .0 & $\mathbf{0}$ & $\mathbf{0}$ & .0 \\
\hline Silver, dissolved (as Ag) & 25 & .0 & .0 & $\mathbf{0}$ & $\mathbf{0}$ & .0 \\
\hline Silver, total recoverable (as Ag) & 27 & .2 & 1.0 & 0 & $\mathbf{5}$ & .2 \\
\hline Strontium, dissolved (as Sr) & 6 & 28.0 & 5.1 & 23 & 37 & 2.1 \\
\hline \multicolumn{7}{|l|}{ Strontium, total } \\
\hline recoverable (as Sr) & 2 & 80.0 & 14.1 & 70 & 90 & 10.0 \\
\hline Vanadium, dissolved (as V) & 6 & 4.2 & 10.2 & $\mathbf{0}$ & 25 & 4.2 \\
\hline Zinc, dissolved (as Zn) & 89 & 12.6 & 16.8 & $\mathbf{0}$ & 100 & 1.8 \\
\hline Zinc, total recoverable (as $\mathrm{Zn}$ ) & 26 & 30.8 & 36.2 & 0 & 190 & 7.1 \\
\hline
\end{tabular}


Table 5.-Selected criteria and the maximum concentration (total recoverable and dissolved) for selected trace elements for each station

(Criteria are from the U.S. Environmental Protection Agency, 1976, unless otherwise noted.)

\begin{tabular}{|c|c|c|c|c|c|c|}
\hline \multirow{2}{*}{$\begin{array}{l}\text { Trace elements } \\
\text { and selected } \\
\text { criteria }\end{array}$} & \multicolumn{6}{|c|}{$\begin{array}{l}\text { Maximum concentration, in micrograms per liter } \\
\text { Total recoverable/dissolved }\end{array}$} \\
\hline & $\begin{array}{l}\text { Blackstone } \\
\text { River at } \\
\text { Millville }\end{array}$ & $\begin{array}{l}\text { Branch } \\
\text { River at } \\
\text { Forestdale }\end{array}$ & $\begin{array}{l}\text { Blackstone } \\
\text { River at } \\
\text { Manville }\end{array}$ & $\begin{array}{l}\text { Pawtuxet } \\
\text { River at } \\
\text { Cranston }\end{array}$ & $\begin{array}{l}\text { Pawtuxet } \\
\text { River at } \\
\text { Pawtuxet }\end{array}$ & $\begin{array}{l}\text { Pawcatuck } \\
\text { River at } \\
\text { Westerly }\end{array}$ \\
\hline $\begin{array}{l}\text { ARSENIC } \\
50 \mu \mathrm{g} / \mathrm{L} \text { for domestic } \\
\text { water supply (health) }\end{array}$ & $6 / 6$ & $1 /-$ & $4 /-$ & $3 /-$ & $4 /-$ & $2 / 2$ \\
\hline $\begin{array}{l}\text { BARIUM } \\
1,000 \mu \mathrm{g} / \mathrm{L} \text { for domestic } \\
\text { water supply (health) }\end{array}$ & $100 / 100$ & $0 /-$ & $0 /-$ & $10 /-$ & $0 /-$ & $100 / 79$ \\
\hline $\begin{array}{l}\text { BORON } \\
750 \mu g / L \text { for long-term irri- } \\
\text { gation on sensitive crops }\end{array}$ & $270 /-$ & $50 /-$ & $240 /-$ & $80 /-$ & $100 /-$ & $80 /-$ \\
\hline $\begin{array}{l}\text { CADMIUM } \\
10 \mu \mathrm{g} / \mathrm{L} \text { for domestic } \\
\text { water supply (health) } \\
0.4 \mu \mathrm{g} / \mathrm{L} \text { for sensitive aquatic } \\
\text { species in soft water } \\
4.0 \mu \mathrm{g} / \mathrm{L} \text { for other, less } \\
\text { sensitive, aquatic species }\end{array}$ & $10 / 8$ & $2 /-$ & $4 /-$ & $4 /-$ & $5 /-$ & $10 / 3$ \\
\hline $\begin{array}{l}\text { CHROMIUM } \\
50 \mu \mathrm{g} / \mathrm{L} \text { for domestic } \\
\text { water supply (health) } \\
100 \mu \mathrm{g} / \mathrm{L} \text { for freshwater } \\
\text { aquatic life }\end{array}$ & $40 / 30$ & $20 /-$ & $50 /-$ & $40 /-$ & $20 /-$ & $30 / 20$ \\
\hline $\begin{array}{l}\text { IRON } \\
300 \mu \mathrm{g} / \mathrm{L} \text { for domestic } \\
\text { water supply (welfare) } \\
1,000 \mu \mathrm{g} / \mathrm{L} \text { for freshwater } \\
\text { aquatic life }\end{array}$ & $1,900 / 420$ & $1,000 / 630$ & $1,500 / 320$ & $860 / 470$ & $1,400 /-$ & $1,200 / 1,100$ \\
\hline $\begin{array}{l}\text { LEAD } \\
50 \mu \mathrm{g} / \mathrm{L} \text { for domestic } \\
\text { water supply (health) }\end{array}$ & $51 / 14$ & $31 /-$ & $45 /-$ & $23 /-$ & $25 /-$ & $34 / 9$ \\
\hline $\begin{array}{l}\text { MANGANESE } \\
50 \mu \mathrm{\mu} / \mathrm{L} \text { for domestic } \\
\text { water supply (welfare) }\end{array}$ & $200 / 230$ & $240 / 0$ & $230 / 260$ & $360 / 130$ & $440 /-$ & $110 / 100$ \\
\hline $\begin{array}{l}\text { MERCURY } \\
2.0 \mu \mathrm{g} / \mathrm{L} \text { for domestic } \\
\text { water supply (health) } \\
0.05 \mathrm{\mu g} / \mathrm{L} \text { for freshwater } \\
\text { aquatic life and wildlife }\end{array}$ & $0.1 / 0.2$ & $0.1 /-$ & $1.8 /-$ & $0.1 /-$ & $0.2 /-$ & $0.3 / 0.7$ \\
\hline $\begin{array}{l}\text { SELENIUM } \\
10 \mu \mathrm{g} / \mathrm{L} \text { for domestic } \\
\text { water supply (health) }\end{array}$ & $1 / 1$ & $2 / 1$ & $1 /-$ & $0 /-$ & $0 /-$ & $0 / 0$ \\
\hline $\begin{array}{l}\text { SILVER } \\
50 \mu g / L \text { for domestic } \\
\text { water supply (health) }\end{array}$ & $1 / 2$ & $0 /-$ & $1 /-$ & $1 /-$ & $71-$ & $5 / 0$ \\
\hline $\begin{array}{l}\text { ZINC } \\
5000 \mu \mathrm{g} / \mathrm{L} \text { for domestic } \\
\text { water supply (welfare) } \\
30 \text { to } 70 \mu \mathrm{g} / \mathrm{L} \text { for sensitive } \\
\text { freshwater aquatic life } \\
\text { (Wentz, 1974, p. 27) }\end{array}$ & $200 / 220$ & $120 / 470$ & $140 /-$ & $130 / 30$ & $120 /-$ & $190 / 100$ \\
\hline
\end{tabular}




\section{Organic Compounds}

A limited number of organic compounds were analyzed and are summarized in table 6. The analyses included the material that was dissolved and the material that was sorbed to suspended particulate matter. Many of these were organochlorine compounds such as DDT, which tend to persist in the environment even though they are no longer used or manufactured.

Of the pesticides, lindane was detected at the Blackstone River at Manville with a maximum concentration of $0.02 \mu \mathrm{g} / \mathrm{L}$ which is less than the drinking water standard of $4 \mathrm{\mu g} / \mathrm{L}$ (U.S. Environmental Protection Agency, 1976). Dieldrin was found at three sites; the Blackstone River at Millville, Mass., the Blackstone River at Manville, and the Pawtuxet River at Cranston. The maximum concentration was $0.01 \mu \mathrm{g} / \mathrm{L}$. PCBs (polychlorinated biphenyls) were found at the same three sites with maximum concentrations of 0.1 , 0.2 , and $2.8 \mu \mathrm{g} / \mathrm{L}$, respectively, and at the Pawtuxet River at Pawtuxet with a maximum concentration of $4.3 \mu \mathrm{g} / \mathrm{L}$.

Phenols (phenolic compounds) include a wide variety of organic chemicals which all have an aromatic ring as the basic chemical structure. Phenols arise from the distillation of coal and wood; from oil refineries; chemical plants; livestock dips; human and other organic wastes; hydrolysis, chemical oxidation, and microbial degradation of pesticides; and from naturally occurring sources and substances. Some compounds strongly resist biological degradation and can be transported long distances in water. Others may be rapidly broken down by biological action which contributes to lowered dissolved oxygen in the stream. The U.S. Environmental Protection Agency (1976) sets a criterion of $1 \mu \mathrm{g} / \mathrm{L}$ for domestic water supply and to protect against tainting of fish flesh. Phenols in excess of this criterion were measured at all six sites. The highest measured concentration was $86 \mu \mathrm{g} / \mathrm{L}$ at Pawtuxet River at Pawtuxet. Phenols were present in each sample collected from this site.

\section{Constituents in Bottom Materials}

Generally, samples of the bottom materials at all six sites were collected during low-flow periods and analyzed for selected trace elements and organic compounds. While there are no standards or criteria for allowable concentrations of these compounds in bottom material, their presence in high concentrations may indicate past or present entry of the materials into the rivers.

Bottom materials often are relatively stationary during periods of low to medium flow. During high flow periods or floods, bottom materials may be resuspended by the higher velocity water and carried downstream where they will be redeposited when the velocity of the water diminishes. In this way, consitutents associated with the bottom material which enter upstream may be distributed downstream.

Table 7 summarizes the bottom material data for the six sites. Data within a site exhibit a wide range in concentration. Bottom materials are seldom distributed uniformly which makes obtaining a "representative" sample or even a duplicate sample difficult. High flows can redistribute bottom material so that another sample collected at the same site at a later time may give entirely different results.

Higher concentrations of elements such as $2,000 \mu \mathrm{g} / \mathrm{g}$ of zinc, $450 \mu \mathrm{g} / \mathrm{g}$ of chromium, and $41,000 \mu \mathrm{g} / \mathrm{g}$ of molybdenum at Blackstone River at Millville, Mass., probably derived from wastes which entered the river some time in the past. Lead values were also elevated at several of the sites.

Organic compounds are often sorbed onto sediment particles and move downstream with the sediments. In the water column, PCBs were found at four of the six sites, dieldrin at three sites, and lindane at one. In the bottom material, chlordane, DDD, DDE, DDT, and PCBs were found at all sites, dieldrin at five sites, and aldrin, endosulfan, endrin, heptachor, and mirex at one or more sites. One high concentration of $\mathrm{PCB}, 5,400 \mu \mathrm{g} / \mathrm{kg}$, was found at Pawtuxet River at Pawtuxet. Subsequent samples contained lower concentrations of PCB. 
Table 6.-Summary of measurements of organic compounds for each station

(Analyses in micrograms per liter except as indicated.)

\begin{tabular}{|c|c|c|c|c|c|c|}
\hline Constituent & $\begin{array}{l}\text { Number } \\
\text { of } \\
\text { samples }\end{array}$ & Mean & $\begin{array}{l}\text { Standard } \\
\text { deviation }\end{array}$ & $\begin{array}{l}\text { Minimum } \\
\text { value }\end{array}$ & $\begin{array}{l}\text { Maximum } \\
\text { value }\end{array}$ & $\begin{array}{c}\text { Standard } \\
\text { error of } \\
\text { mean }\end{array}$ \\
\hline
\end{tabular}

01111230 Blackstone River at Millville, Mass.

Carbon, organic total

(mg/L as C)

Phenols, total

Methylene blue active

substance $(\mathrm{mg} / \mathrm{L}$ )

Oil and grease, total recoverable extraction

gravimetric $(\mathrm{mg} / \mathrm{L})$

PCB, total

Napthalenes, polychlorinated, total

Aldrin, total

Chlordane, total

DDD, total

DDE, total

DDT, total

Dieldrin, total

Endosulfan, total

Endrin, total

Heptachlor, total

Heptachlor epoxide, total

Lindane, total

Methoxychlor, total

Mirex, total

Perthane, total

Toxaphene, total
24

14

5

.160

.9

.02

6

6

6

6

6

6

6

6

6

6

6

6

6

6

5

5

6

6

6.67

1.4
.134

1.5

.04

.00

.00

.00

.00

.00

.00

.005

.00

.00

.00

.00

.00

.00

.00

.00

.00
3.8

0

.10

.40

.060

0.39

.4

5
.4

.02

.0

.1

.00

.00

.00

.00

.00

.00

.002

.00

.00

.00

.00

.00

.00

.00

.00

.00 
Table 6.-Summary of measurements of organic compounds for each station (continued)

\begin{tabular}{|c|c|c|c|c|c|c|}
\hline Constituent & $\begin{array}{l}\text { Number } \\
\text { of } \\
\text { samples }\end{array}$ & Mean & $\begin{array}{l}\text { Standard } \\
\text { deviation }\end{array}$ & $\begin{array}{c}\text { Minimum } \\
\text { value }\end{array}$ & $\begin{array}{l}\text { Maximum } \\
\text { value }\end{array}$ & $\begin{array}{l}\text { Standard } \\
\text { error of } \\
\text { mean }\end{array}$ \\
\hline
\end{tabular}

01111500 Branch River at Forestdale, R. I.

Carbon, organic total$$
\text { (mg/L as C) }
$$

Phenols, total

Methylene blue active substance ( $\mathrm{mg} / \mathrm{L}$ )

Oil and grease, total recoverable extraction gravimetric (mg/L)

PCB, total

Napthalenes, polychlorinated, total

Aldrin, total

Chlordane, total

DDD, total

DDE, total

DDT, total

Dieldrin, total

Endosulfan, total

Endrin, total

Heptachlor, total

Heptachlor epoxide, total

Lindane, total

Methoxychlor, total

Mirex, total

Perthane, total

Toxaphene, total
12

14

9

5.71

1.4

.000

16

6

.4

.00

1.0

.00

.00

.00

.00

.00

.00

.00

.00

.00

.00

.00

.00

.00

.00

.00

.00

.00
3.9

0

.00

.00

.000

.000

.

0

.0

4

.3

.00

.00

.00

.00

.00

.00

.00

.00

.00

.00

.00

.00

.00

.00

.00

.00

.00 
Table 6.-Summary of measure ments of organic compounds for each station (continued)

\begin{tabular}{|c|c|c|c|c|c|c|}
\hline Constituent & $\begin{array}{l}\text { Number } \\
\text { of } \\
\text { samples }\end{array}$ & Mean & $\begin{array}{l}\text { Standard } \\
\text { deviation }\end{array}$ & $\begin{array}{l}\text { Minimum } \\
\text { value }\end{array}$ & $\begin{array}{l}\text { Maximum } \\
\text { value }\end{array}$ & $\begin{array}{c}\text { Standard } \\
\text { error of } \\
\text { mean }\end{array}$ \\
\hline & \multicolumn{5}{|c|}{01112900 Blackstone River at Manville, R. I. } & \\
\hline \multicolumn{7}{|l|}{ Carbon, organic total } \\
\hline (mg/L as C) & 12 & 6.82 & 1.59 & 5.0 & 9.7 & 0.46 \\
\hline Phenols, total & 16 & 5.6 & 11.2 & $\mathbf{0}$ & 45 & 2.8 \\
\hline \multicolumn{7}{|l|}{ Methylene blue active } \\
\hline substance $(\mathrm{mg} / \mathrm{L})$ & 10 & .130 & .067 & .10 & .30 & .021 \\
\hline \multicolumn{7}{|l|}{ Oil and grease, total } \\
\hline gravimetric (mg/L) & 17 & .6 & 1.0 & $\mathbf{0}$ & 3 & .2 \\
\hline PCB, total & $\mathbf{5}$ & .08 & .11 & .0 & .2 & .05 \\
\hline \multicolumn{7}{|l|}{ Napthalenes, polychlor- } \\
\hline inated, total & 5 & .00 & .00 & .0 & .0 & .00 \\
\hline Aldrin, total & 5 & .00 & .00 & .0 & .0 & .00 \\
\hline Chlordane, total & 5 & .00 & .00 & .0 & .0 & .00 \\
\hline $\mathrm{DDD}$, total & 5 & .00 & .00 & .0 & .0 & .00 \\
\hline DDE, total & $\mathbf{5}$ & .00 & .00 & .0 & .0 & .00 \\
\hline DDT, total & 5 & .00 & .00 & .0 & .0 & .00 \\
\hline Dieldrin, total & 5 & .006 & .005 & .00 & .01 & .002 \\
\hline Endosulfan, total & 5 & .00 & .00 & .0 & .0 & .00 \\
\hline Endrin, total & $\mathbf{5}$ & .00 & .00 & .0 & .0 & .00 \\
\hline Heptachlor, total & 5 & .00 & .00 & .0 & .0 & .00 \\
\hline Heptachlor epoxide, total & 5 & .00 & .00 & .0 & .0 & .00 \\
\hline Lindane, total & 5 & .004 & .009 & .00 & .02 & .004 \\
\hline Methoxychlor, total & $\mathbf{5}$ & .00 & .00 & .0 & .0 & .00 \\
\hline Mirex, total & 5 & .00 & .00 & .0 & .0 & .00 \\
\hline Perthane, total & 5 & .00 & .00 & .0 & .0 & .00 \\
\hline Toxaphene, total & 5 & .00 & .00 & .0 & .0 & .00 \\
\hline
\end{tabular}


Table 6.-Summary of measurements of organic compounds for each station (continued)

\begin{tabular}{|c|c|c|c|c|c|c|}
\hline Constituent & $\begin{array}{c}\text { Number } \\
\text { of } \\
\text { samples }\end{array}$ & Mean & $\begin{array}{l}\text { Standard } \\
\text { deviation }\end{array}$ & $\begin{array}{c}\text { Minimum } \\
\text { value }\end{array}$ & $\begin{array}{l}\text { Maximum } \\
\text { value }\end{array}$ & $\begin{array}{c}\text { Standard } \\
\text { error of } \\
\text { mean }\end{array}$ \\
\hline
\end{tabular}

01116500 Pawtuxet River at Cranston, R. I.

Carbon, organic total

$$
\text { (mg/L as C) }
$$

Phenols, total

Methylene blue active substance (mg/L)

Oil and grease, total recoverable extraction gravimetric (mg/L)

PCB, total

Napthalenes, polychlorinated, total

Aldrin, total

Chlordane, total

DDD, total

DDE, total

DDT, total

Dieldrin, total

Endosulfan, total

Endrin, total

Heptachlor, total

Heptachlor epoxide, total

Lindane, total

Methoxychlor, total

Mirex, total

Perthane, total

Toxaphene, total
12

19

10

17

8

1.1

.64

8

8

8

8

8

7

8

8

8

8

5

5

7

8

.00

.00

.00
2.58

4.7

.052

1.7

1.09

.00

.00

.00

.00

.00

.00

.004

.00

.00

.00

.00

.00

.00

.00

.00

.00
4.6

0

.10

.20
0.75

1.1

15
0

.0

.0

.0

.0

.0

.0

.0

.00

.0

.0

.0

.0

.0

.0

.0

.0

.0
6

2.8

.4

.38

.00

.00

.00

.00

.00

.00

.001

.00

.00

.00

.00

.00

.00

.00

.00

.00 
Table 6.-Summary of measurements of organic compounds for each station (continued)

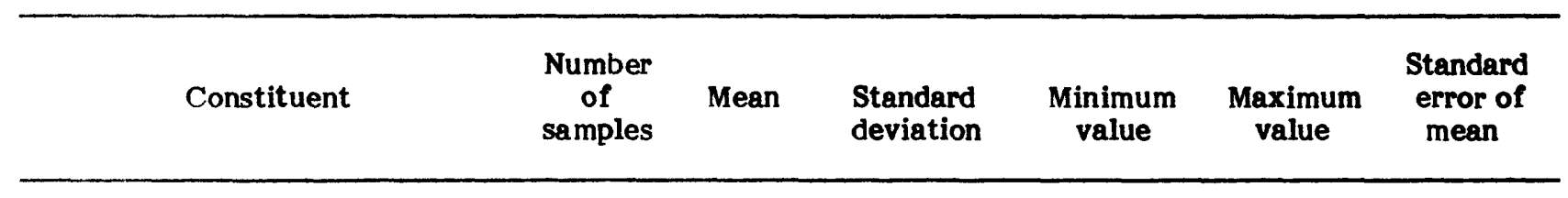

01116617 Pawtuxet River at Pawtuxet, R. I.

Carbon, organic total

(mg/L as C)

12

15

Phenols, total

Methylene blue active

substance (mg/L)

Oil and grease, total

recoverable extraction

gravimetric (mg/L)

PCB, total

Napthalenes, polychlorinated, total

Aldrin, total

Chlordane, total

DDD, total

DDE, total

DDT, total

Dieldrin, total

Endosulfan, total

Endrin, total

Heptachlor, total

Heptachlor epoxide, total

Lindane, total

Methoxychlor, total

Mirex, total

Perthane, total

Toxaphene, total

10

12

$$
10.96
$$

31.5

.180

4.49

27.8

.042

.10

.20

1.30

7.2

1

86

.013

$\begin{array}{rccccc}16 & .6 & .9 & 0 & 3 & .2 \\ 4 & 1.48 & 2.03 & .0 & 4.3 & 1.01\end{array}$$$
4.00
$$$$
4 \quad .00
$$$$
4 \quad .00
$$$$
4 \quad .00
$$$$
4 \quad .00
$$$$
4 \quad .00
$$

$4 \quad .00$

$4 \quad .00$

$4 \quad .00$

$4 \quad .00$

$3 \quad .00$

$3 \quad .00$

$4 \quad .00$

4

.00
.00

.00

.00

.00

.00

.00

.00

.00

.00

.00

.00

.00

.00

.00

.00

.00

$\begin{array}{lll}.0 & .0 & .00 \\ .0 & .0 & .00 \\ .0 & .0 & .00 \\ .0 & .0 & .00 \\ .0 & .0 & .00 \\ .0 & .0 & .00 \\ .0 & .0 & .00 \\ .0 & .0 & .00 \\ .0 & .0 & .00 \\ .0 & .0 & .00 \\ .0 & .0 & .00 \\ .0 & .0 & .00 \\ .0 & .0 & .00 \\ .0 & .0 & .00 \\ .0 & .0 & .00 \\ .0 & .0 & .00\end{array}$


Table 6.-Summary of measurements of organic compounds for each station (continued)

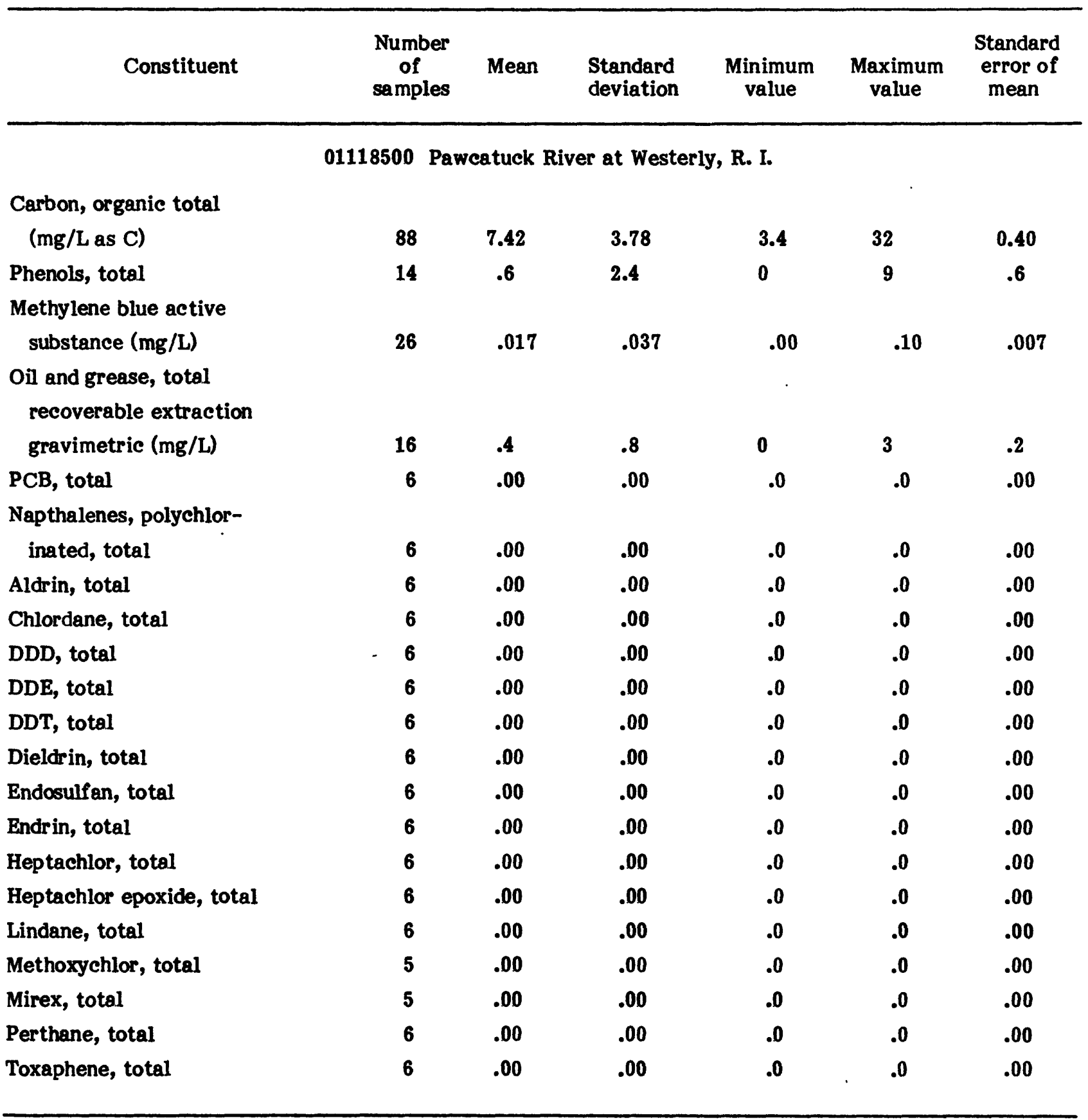


Table 7.-Summary of measurements of constituents in the bottom material for each station

\begin{tabular}{|c|c|c|c|c|c|c|}
\hline Constituent & $\begin{array}{l}\text { Number } \\
\text { of } \\
\text { samples }\end{array}$ & Mean & $\begin{array}{l}\text { Standard } \\
\text { deviation }\end{array}$ & $\begin{array}{l}\text { Minimum } \\
\text { value }\end{array}$ & $\begin{array}{l}\text { Maximum } \\
\text { value }\end{array}$ & $\begin{array}{l}\text { Standard } \\
\text { error of } \\
\text { mean }\end{array}$ \\
\hline
\end{tabular}

01111230 Blackstone River at Millville, Mass.

(The following analyses are in micrograms per gram except as indicated.)

C.O.D., total in bottom material ( $\mathrm{mg} / \mathrm{kg})$

Aluminum, recoverable from bottom material

Arsenic, total in bottom material

Barium, recoverable from bottom material

Beryllium, recoverable from bottom material

Boron, recoverable from bottom material

Cadmium, recoverable from bottom material

Chromium, recoverable from bottom material

Cobalt, recoverable from bottom material

Copper, recoverable from bottom material

Iron, recoverable from bottom material

Lead, recoverable from bot tom material

Manganese, recoverable from bottom material

Mercury, recoverable from bottom material

Molybdenum, recoverable from bottom material

Nickel, recoverable from bottom material

Selenium, total in bottom material

Silver, recoverable from bottom material

Strontium, recoverable from bottom material

Vanadium, total in bottom material

Zinc, recoverable from bottom material

1

11

23,900

36,000

6,650

1.3

7

$$
.7
$$

130

156

2

.0

.0

$$
.0
$$

19.0

30.3

168

127

5.7

15.1

230

163

230

7

11,600

14,700

93.0

4,600

130,000

10,900

1,600

11,000

4,700

3

.5

240

110$$
0
$$

o

$$
0
$$

80

450

10

o

40

640

44,000

260

823

.34

2,700

38

40

2,300

.0

.9

41,000

200

13,700

313,700

23,700

86.1

0

0

0

1.8

42.4

10

40.0

$-$

716

40

2,000

270
11.5

63.7

5.7

86.9

5,550

35.2

311

.13

38.5

$$
.2
$$

.8

30.0

\section{.0}

.0


Table 7.-Summary of measurements of constituents in the bottom material for each station (continued)

\begin{tabular}{|c|c|c|c|c|c|c|}
\hline Constituent & $\begin{array}{l}\text { Number } \\
\text { of } \\
\text { samples }\end{array}$ & Mean & $\begin{array}{l}\text { Standard } \\
\text { deviation }\end{array}$ & $\begin{array}{l}\text { Minimum } \\
\text { value }\end{array}$ & $\begin{array}{l}\text { Maximum } \\
\text { value }\end{array}$ & $\begin{array}{l}\text { Standard } \\
\text { error of } \\
\text { mean }\end{array}$ \\
\hline
\end{tabular}

01111230 Blackstone River at Millville, Mass. (continued)

(The following analyses are in micrograms per kilogram.)

\begin{tabular}{|c|c|c|c|c|c|c|}
\hline $\begin{array}{l}\text { Aldrin, total in } \\
\text { bottom material }\end{array}$ & 6 & 0.00 & 0.00 & 0.0 & 0.0 & 0.00 \\
\hline $\begin{array}{l}\text { Chlordane, total in } \\
\text { bottom material }\end{array}$ & 6 & 16.5 & 21.4 & 2.0 & 55 & 8.74 \\
\hline $\begin{array}{l}\text { DDD, total in } \\
\text { bottom material }\end{array}$ & 6 & 8.91 & 10.6 & 1.3 & 27 & 4.35 \\
\hline $\begin{array}{l}\text { DDE, total in } \\
\text { bottom material }\end{array}$ & 6 & .68 & 1.06 & .0 & 2.1 & .43 \\
\hline $\begin{array}{l}\text { DDT, total in } \\
\text { bottom material }\end{array}$ & 6 & 1.32 & 1.41 & .0 & 4.0 & .57 \\
\hline $\begin{array}{l}\text { Dieldrin, total in } \\
\text { bottom material }\end{array}$ & 6 & 78.9 & 177 & 2.7 & 440 & 72.3 \\
\hline $\begin{array}{l}\text { Endosulfan, total in } \\
\text { bottom material }\end{array}$ & 4 & .00 & .00 & .0 & .0 & .00 \\
\hline $\begin{array}{l}\text { Endrin, total in } \\
\text { bottom material }\end{array}$ & 6 & .00 & .00 & .0 & .0 & .00 \\
\hline $\begin{array}{l}\text { Heptachlor, total in } \\
\text { bottom material }\end{array}$ & 6 & .05 & .12 & .0 & .3 & .05 \\
\hline $\begin{array}{l}\text { Heptachlor epoxide, total } \\
\text { in bottom material }\end{array}$ & 6 & .00 & .00 & .0 & .0 & .00 \\
\hline $\begin{array}{l}\text { Lindane, total in } \\
\text { bottom material }\end{array}$ & 6 & .00 & .00 & .0 & .0 & .00 \\
\hline $\begin{array}{l}\text { Methoxychlor, total in } \\
\text { bottom material }\end{array}$ & 6 & .00 & .00 & .0 & .0 & .00 \\
\hline $\begin{array}{l}\text { Mirex, total in } \\
\text { bottom material }\end{array}$ & 4 & .00 & .00 & .0 & .0 & .00 \\
\hline $\begin{array}{l}\mathrm{PCB} \text {, total in } \\
\text { bottom material }\end{array}$ & 6 & 101 & 153 & .0 & 410 & 62.5 \\
\hline $\begin{array}{l}\text { PCN, total in } \\
\text { bottom material }\end{array}$ & 4 & .00 & .00 & .0 & .00 & .00 \\
\hline $\begin{array}{l}\text { Perthane, total in } \\
\text { bottom material }\end{array}$ & 4 & .00 & .00 & .0 & .00 & .00 \\
\hline $\begin{array}{l}\text { Toxaphene, total in } \\
\text { bottom material }\end{array}$ & 6 & .00 & .00 & .0 & .00 & .00 \\
\hline
\end{tabular}


Table 7.-Summary of measurements of constituents in the bottom material for each station (continued)

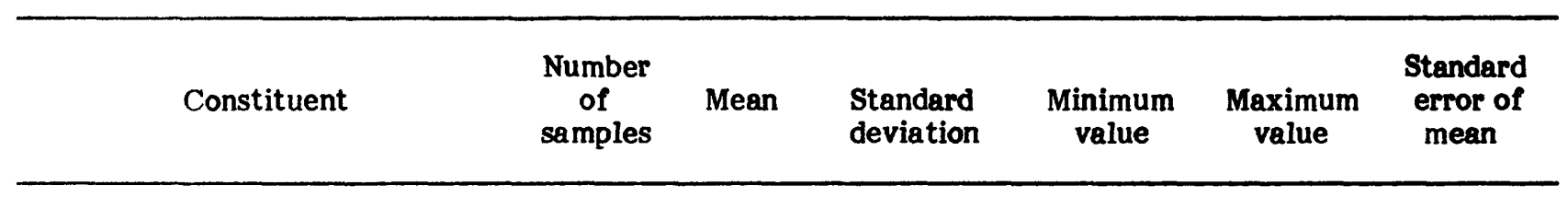

01111500 Branch River at Forestdale, R. I.

(The following analyses are in micrograms per gram except as indicated.)

C.O.D., total in bottom material (mg/kg)

Aluminum, recoverable from bottom material

Arsenic, total in bottom material

Barium, recoverable from bottom material

Beryllium, recoverable from bottom material

Boron, recoverable from bottom material

Cadmium, recoverable from bottom material

Chromium, recoverable from bottom material

Cobalt, recoverable from bottom material

Copper, recoverable from bottom material

Iron, recoverable from bottom material

Lead, recoverable from bottom material

Manganese, recoverable from bottom material

Mercury, recoverable from bottom material

Molybdenum, recoverable from bottom material

Nickel, recoverable from bottom material

Selenium, total in bottom material

Silver, recoverable from bottom material

Strontium, recoverable from bottom material

Vanadium, total in bottom material

Zinc, recoverable from bottom material

\begin{tabular}{|c|c|c|c|c|c|}
\hline 11 & 36,400 & 31,800 & 2,000 & 120,000 & 9,590 \\
\hline 2 & 4,450 & 3,460 & 2,000 & 6,900 & 2,450 \\
\hline 7 & .1 & .4 & 0 & 10 & .1 \\
\hline 2 & 45 & 35 & 20 & 70 & 25 \\
\hline 2 & .0 & .0 & 0 & 0 & .0 \\
\hline 5 & 4.0 & 8.9 & 0 & 20 & 4.0 \\
\hline 7 & 4.6 & 7.4 & 0 & 20 & 2.8 \\
\hline 7 & 24.3 & 17.2 & 0 & 50 & 6.5 \\
\hline 7 & .0 & .0 & 0 & 0 & .0 \\
\hline 7 & 55.7 & 58.8 & 0 & 180 & 22.2 \\
\hline 7 & 5,040 & 3,390 & 1,800 & 11,000 & 1,280 \\
\hline 7 & 43.0 & 21.3 & 20 & 70 & 8.1 \\
\hline 7 & 155 & 117 & 30 & 370 & 44.3 \\
\hline 7 & .02 & .02 & .0 & .1 & .01 \\
\hline 2 & .0 & .0 & 0 & 0 & .0 \\
\hline 7 & 14.1 & 19.8 & 0 & 50 & 7.5 \\
\hline 7 & .4 & .8 & 0 & 2 & .3 \\
\hline 6 & .0 & .0 & 0 & 0 & .0 \\
\hline 2 & 20.0 & 14.1 & 10 & 30 & 10.0 \\
\hline 0 & - & - & - & - & - \\
\hline 7 & 135 & 131 & 20 & 400 & 49.5 \\
\hline
\end{tabular}

49.5 
Table 7.-Summary of measurements of constituents in the bottom material for each station (continued)

\begin{tabular}{|c|c|c|c|c|c|c|}
\hline Constituent & $\begin{array}{l}\text { Number } \\
\text { of } \\
\text { samples }\end{array}$ & Mean & $\begin{array}{l}\text { Standard } \\
\text { deviation }\end{array}$ & $\begin{array}{l}\text { Minimum } \\
\text { value }\end{array}$ & $\begin{array}{l}\text { Maximum } \\
\text { value }\end{array}$ & $\begin{array}{l}\text { Standard } \\
\text { error of } \\
\text { mean }\end{array}$ \\
\hline
\end{tabular}

01111500 Branch River at Forestdale, R. I. (continued)

(The following analyses are in micrograms per kilogram.)

\begin{tabular}{|c|c|c|c|c|c|c|}
\hline $\begin{array}{l}\text { Aldrin, total in } \\
\text { bottom material }\end{array}$ & 6 & 0.00 & 0.00 & 0.0 & 0.0 & 0.00 \\
\hline $\begin{array}{l}\text { Chlordane, total in } \\
\text { bottom material }\end{array}$ & 6 & 15.8 & 17.9 & .0 & 49 & 7.29 \\
\hline $\begin{array}{l}\text { DDD, total in } \\
\text { bottom material }\end{array}$ & 6 & 14.2 & 18.4 & .0 & 48 & 7.50 \\
\hline $\begin{array}{l}\mathrm{DDE} \text {, total in } \\
\text { bottom material }\end{array}$ & 6 & .95 & 2.05 & .0 & 5.1 & .84 \\
\hline $\begin{array}{l}\text { DDT, total in } \\
\text { bottom material }\end{array}$ & 6 & 3.27 & 5.18 & .0 & 13 & 2.11 \\
\hline $\begin{array}{l}\text { Dieldrin, total in } \\
\text { bottom material }\end{array}$ & 6 & .35 & .64 & .0 & 1.6 & .26 \\
\hline $\begin{array}{l}\text { Endosulfan, total in } \\
\text { bottom material }\end{array}$ & 4 & .00 & .00 & .0 & .0 & .00 \\
\hline $\begin{array}{l}\text { Endrin, total in } \\
\text { bottom material }\end{array}$ & 6 & .00 & .00 & .0 & .0 & .00 \\
\hline $\begin{array}{l}\text { Heptachlor, total in } \\
\text { bottom material }\end{array}$ & 6 & .00 & .00 & .0 & .0 & .00 \\
\hline $\begin{array}{l}\text { Heptachlor epoxide, total } \\
\text { in bottom material }\end{array}$ & 6 & .00 & .00 & .0 & .0 & .00 \\
\hline $\begin{array}{l}\text { Lindane, total in } \\
\text { bottom material }\end{array}$ & 6 & .00 & .00 & .0 & .0 & .00 \\
\hline $\begin{array}{l}\text { Methoxychlor, total in } \\
\text { bottom material }\end{array}$ & 6 & .00 & .00 & .0 & .0 & .00 \\
\hline $\begin{array}{l}\text { Mirex, total in } \\
\text { bottom material }\end{array}$ & 4 & 109 & 145 & .0 & 310 & 72.6 \\
\hline $\begin{array}{l}\text { PCB, total in } \\
\text { bottom material }\end{array}$ & 6 & 114 & 234 & 3.0 & 590 & 95.3 \\
\hline $\begin{array}{l}\text { PCN, total in } \\
\text { bottom material }\end{array}$ & 4 & .00 & .00 & .0 & .0 & .00 \\
\hline $\begin{array}{l}\text { Perthane, total in } \\
\text { bottom material }\end{array}$ & 4 & .00 & .00 & .0 & .0 & .00 \\
\hline $\begin{array}{l}\text { Toxaphene, total in } \\
\text { bottom material }\end{array}$ & 6 & .00 & .00 & .0 & .0 & .00 \\
\hline
\end{tabular}


Table 7.-Summary of measurements of constituents in the bottom material for each station (continued)

\begin{tabular}{|c|c|c|c|c|c|c|}
\hline Constituent & $\begin{array}{l}\text { Number } \\
\text { of } \\
\text { samples }\end{array}$ & Mean & $\begin{array}{l}\text { Standard } \\
\text { deviation }\end{array}$ & $\begin{array}{l}\text { Minimum } \\
\text { value }\end{array}$ & $\begin{array}{l}\text { Maximum } \\
\text { value }\end{array}$ & $\begin{array}{l}\text { Standard } \\
\text { error of } \\
\text { mean }\end{array}$ \\
\hline
\end{tabular}

01112900 Blackstone River at Manville, R. I.

(The following analyses are in micrograms per gram except as indicated.)

C.O.D., total in bottom material ( $\mathrm{mg} / \mathrm{kg}$ )

Aluminum, recoverable from bottom material

Arsenic, total in bottom material

Barium, recoverable from bottom material

Beryllium, recoverable from bottom material

Boron, recoverable from bottom material

Cadmium, recoverable from bottom material

Chromium, recoverable from bottom material

Cobalt, recoverable from bottom material

Copper, recoverable from bottom material

Iron, recoverable from bottom material

Lead, recoverable from bottom material

Manganese, recoverable from bottom material

Mercury, recoverable from bottom material

Molybdenum, recoverable from bottom material

Nickel, recoverable from bottom material

Selenium, total in bottom material

Silver, recoverable from bottom material

Strontium, recoverable from bottom material

Vanadium, total in bottom material

Zine, recoverable from bottom material

11

11

$$
25,600 \quad 41,700
$$

1

1700

6

.0

1

40

2

5

6

6

6

6

6

6

6

6

1

6

6

5

1

.0

4.0

1.0

13.3

1.7

$6 \quad 29.0$

10.5

8.9

1.7

8.2

4.1

4,400

$\cdot 150,000$

12,600

1700

1700

.0

0

0

.0

40

0

0

40

0

20

4.0

4.1

3,280

1,400

18.6

44.3

21.0

23

30

.02

.0

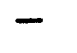

8.4

5.0

.0

.0

. 0

.0

20.0

$-$

0

6

400

784

\section{4}

20

10

40

5,700

70

80

.1

8.6

.01

o

0

0

20

3.4

.0

.0

$\begin{array}{lll}0 & 0 & .0\end{array}$

1

-
20

30

2,000 
Table 7.-Summary of measurements of constituents in the bottom material for each station (continued)

\begin{tabular}{|c|c|c|c|c|c|c|}
\hline Constituent & $\begin{array}{l}\text { Number } \\
\text { of } \\
\text { samples }\end{array}$ & Mean & $\begin{array}{l}\text { Standard } \\
\text { deviation }\end{array}$ & $\begin{array}{l}\text { Minimum } \\
\text { value }\end{array}$ & $\begin{array}{l}\text { Maximum } \\
\text { value }\end{array}$ & $\begin{array}{l}\text { Standard } \\
\text { error of } \\
\text { mean }\end{array}$ \\
\hline
\end{tabular}

01112900 Blackstone River at Manville, R. I. (continued)

(The following analyses are in micrograms per kilogram.)

\begin{tabular}{|c|c|c|c|c|c|c|}
\hline $\begin{array}{l}\text { Aldrin, total in } \\
\text { bottom material }\end{array}$ & 6 & 0.00 & 0.00 & 0.0 & 0.0 & 0.00 \\
\hline $\begin{array}{c}\text { Chlordane, total in } \\
\text { bottom material }\end{array}$ & 6 & 20.2 & 19.6 & .0 & 50 & 8.01 \\
\hline $\begin{array}{l}\mathrm{DDD}, \text { total in } \\
\text { bottom material }\end{array}$ & 6 & 18.0 & 22.7 & .0 & 48 & 9.25 \\
\hline $\begin{array}{l}\mathrm{DDE} \text {, total in } \\
\text { bottom material }\end{array}$ & 6 & 5.38 & 8.40 & .0 & 22 & 3.43 \\
\hline $\begin{array}{l}\text { DDT, total in } \\
\text { bottom material }\end{array}$ & 6 & 1.68 & 1.93 & .0 & 5.0 & .79 \\
\hline $\begin{array}{l}\text { Dieldrin, total in } \\
\text { bottom material }\end{array}$ & 6 & 11.6 & 14.5 & .0 & 35 & 5.91 \\
\hline $\begin{array}{l}\text { Endosulfan, total in } \\
\text { bottom material }\end{array}$ & 4 & .00 & .00 & .0 & .0 & .00 \\
\hline $\begin{array}{l}\text { Endrin, total in } \\
\text { bottom material }\end{array}$ & 6 & .00 & .00 & .0 & .0 & .00 \\
\hline $\begin{array}{l}\text { Heptachlor, total in } \\
\text { bottom material }\end{array}$ & 6 & .02 & .04 & .0 & .1 & .02 \\
\hline $\begin{array}{l}\text { Heptachlor epoxide, total } \\
\text { in bottom material }\end{array}$ & 6 & .00 & .00 & .0 & .0 & .00 \\
\hline $\begin{array}{l}\text { Lindane, total in } \\
\text { bottom material }\end{array}$ & 6 & .00 & .00 & .0 & .0 & .00 \\
\hline $\begin{array}{l}\text { Methoxychlor, total in } \\
\text { bottom material }\end{array}$ & 6 & .00 & .00 & .0 & .0 & .00 \\
\hline $\begin{array}{l}\text { Mirex, total in } \\
\text { bottom material }\end{array}$ & 4 & .00 & .00 & .0 & .0 & .00 \\
\hline $\begin{array}{l}\text { PCB, total in } \\
\text { bottom material }\end{array}$ & 6 & 117 & 98.1 & 26 & 280 & 40.1 \\
\hline $\begin{array}{l}\mathrm{PCN}, \text { total in } \\
\text { bottom material }\end{array}$ & 4 & .00 & .00 & .0 & .0 & .00 \\
\hline $\begin{array}{l}\text { Perthane, total in } \\
\text { bottom material }\end{array}$ & 4 & .00 & .00 & .0 & .0 & .00 \\
\hline $\begin{array}{l}\text { Toxaphene, total in } \\
\text { bottom material }\end{array}$ & 6 & .00 & .00 & .0 & .0 & .00 \\
\hline
\end{tabular}


Table 7.-Summary of measurements of constituents in the bottom material for each station (continued)

\begin{tabular}{|c|c|c|c|c|c|c|}
\hline Constituent & $\begin{array}{l}\text { Number } \\
\text { of } \\
\text { samples }\end{array}$ & Mean & $\begin{array}{l}\text { Standard } \\
\text { deviation }\end{array}$ & $\begin{array}{l}\text { Minimum } \\
\text { value }\end{array}$ & $\begin{array}{l}\text { Maximum } \\
\text { value }\end{array}$ & $\begin{array}{l}\text { Standard } \\
\text { error of } \\
\text { mean }\end{array}$ \\
\hline
\end{tabular}

01116500 Pawtuxet River at Cranston, R. I.

(The following analyses are in micrograms per gram except as indicated.)

\begin{tabular}{|c|c|c|c|c|c|c|}
\hline $\begin{array}{l}\text { C.O.D., total in bottom } \\
\text { material (mg/kg) }\end{array}$ & 17 & 12,800 & 12,000 & 1,700 & 47,000 & 2,920 \\
\hline $\begin{array}{l}\text { Aluminum, recoverable from } \\
\text { bottom material }\end{array}$ & 3 & 2,100 & 1,060 & 900 & 2,900 & 611 \\
\hline $\begin{array}{l}\text { Arsenic, total in } \\
\text { bottom material }\end{array}$ & 7 & .0 & .0 & 0 & 0 & .0 \\
\hline $\begin{array}{l}\text { Barium, recoverable from } \\
\text { bottom material }\end{array}$ & 3 & 23 & 16 & 8 & 40 & 9.3 \\
\hline $\begin{array}{l}\text { Beryllium, recoverable from } \\
\text { bottom material }\end{array}$ & 3 & .0 & .0 & 0 & 00 & .0 \\
\hline $\begin{array}{l}\text { Boron, recoverable from } \\
\text { bottom material }\end{array}$ & 6 & 3.7 & 8.0 & 0 & 20 & 3.3 \\
\hline $\begin{array}{l}\text { Cadmium, recoverable from } \\
\text { bottom material }\end{array}$ & 7 & .7 & 1.3 & 0 & 3 & .5 \\
\hline $\begin{array}{l}\text { Chromium, recoverable from } \\
\text { bottom material }\end{array}$ & 12 & 5.5 & 6.5 & 0 & 20 & 1.9 \\
\hline $\begin{array}{l}\text { Cobalt, recoverable from } \\
\text { bottom material }\end{array}$ & 8 & .1 & .4 & 0 & 1 & .1 \\
\hline $\begin{array}{l}\text { Copper, recoverable from } \\
\text { bottom material }\end{array}$ & 8 & 36.9 & 32.9 & 10 & 90 & 11.6 \\
\hline $\begin{array}{l}\text { Iron, recoverable from } \\
\text { bottom material }\end{array}$ & 8 & 4,410 & 2,170 & 900 & 7,000 & 769 \\
\hline $\begin{array}{l}\text { Lead, recoverable from } \\
\text { bottom material }\end{array}$ & 8 & 67.0 & 94.2 & 10 & 290 & 33.3 \\
\hline $\begin{array}{l}\text { Manganese, recoverable from } \\
\text { bottom material }\end{array}$ & 8 & 76.5 & 36.1 & 23 & 140 & 12.8 \\
\hline $\begin{array}{l}\text { Mercury, recoverable from } \\
\text { bottom material }\end{array}$ & 7 & .05 & .12 & .0 & .3 & .04 \\
\hline $\begin{array}{l}\text { Molybdenum, recoverable from } \\
\text { bottom material }\end{array}$ & 3 & .0 & .0 & 0 & 0 & .0 \\
\hline $\begin{array}{l}\text { Nickel, recoverable from } \\
\text { bottom material }\end{array}$ & 8 & 6.0 & 8.5 & 0 & 20 & 3.0 \\
\hline $\begin{array}{l}\text { Selenium, total in } \\
\text { bottom material }\end{array}$ & 7 & .0 & .0 & 0 & 0 & .0 \\
\hline $\begin{array}{l}\text { Silver, recoverable from } \\
\text { bottom material }\end{array}$ & 7 & .1 & .4 & 0 & 1 & .1 \\
\hline $\begin{array}{l}\text { Strontium, recoverable from } \\
\text { bottom material }\end{array}$ & 3 & 7.0 & 5.2 & 1 & 10 & 3.0 \\
\hline $\begin{array}{l}\text { Vanadium, total in } \\
\text { bottom material }\end{array}$ & 1 & 9.0 & - & 9 & 9 & - \\
\hline $\begin{array}{l}\text { Zinc, recoverable from } \\
\text { bottom material }\end{array}$ & 7 & 41.6 & 25.7 & 18 & 90 & 9.7 \\
\hline
\end{tabular}


Table 7.-Summary of measurements of constituents in the bottom material for each station (continued)

\begin{tabular}{|c|c|c|c|c|c|c|}
\hline Constituent & $\begin{array}{l}\text { Number } \\
\text { of } \\
\text { samples }\end{array}$ & Mean & $\begin{array}{l}\text { Standard } \\
\text { deviation }\end{array}$ & $\begin{array}{l}\text { Minimum } \\
\text { value }\end{array}$ & $\begin{array}{l}\text { Maximum } \\
\text { value }\end{array}$ & $\begin{array}{c}\text { Standard } \\
\text { error of } \\
\text { mean }\end{array}$ \\
\hline
\end{tabular}

01116500 Pawtuxet River at Cranston, R. I. (continued)

(The following analyses are in micrograms per kilogram.)

\begin{tabular}{|c|c|c|c|c|c|c|}
\hline $\begin{array}{l}\text { Aldrin, total in } \\
\text { bottom material }\end{array}$ & 6 & 0.17 & 0.41 & 0.0 & 1.0 & 0.17 \\
\hline $\begin{array}{l}\text { Chlordane, total in } \\
\text { bottom material }\end{array}$ & 6 & 5.00 & 2.61 & 3.0 & 10 & 1.06 \\
\hline $\begin{array}{l}\text { DDD, total in } \\
\text { bottom material }\end{array}$ & 6 & 3.32 & 6.30 & .0 & 16 & 2.57 \\
\hline $\begin{array}{l}\text { DDE, total in } \\
\text { bottom material }\end{array}$ & 6 & .73 & .96 & .0 & 2.4 & .39 \\
\hline $\begin{array}{l}\text { DDT, total in } \\
\text { bottom material }\end{array}$ & 6 & .55 & .84 & .0 & 2.1 & .34 \\
\hline $\begin{array}{l}\text { Dieldrin, total in } \\
\text { bottom material }\end{array}$ & 6 & .05 & .08 & .0 & .2 & .03 \\
\hline $\begin{array}{l}\text { Endosulfan, total in } \\
\text { bottom material }\end{array}$ & 4 & .00 & .00 & .0 & .0 & .00 \\
\hline $\begin{array}{l}\text { Endrin, total in } \\
\text { bottom material }\end{array}$ & 6 & .00 & .00 & .0 & .0 & .00 \\
\hline $\begin{array}{l}\text { Heptachlor, total in } \\
\text { bottom material }\end{array}$ & 6 & .08 & .20 & .0 & .5 & .08 \\
\hline $\begin{array}{l}\text { Heptachlor epoxide, total } \\
\text { in bottom material }\end{array}$ & 6 & .00 & .00 & .0 & .0 & .00 \\
\hline $\begin{array}{l}\text { Lindane, total in } \\
\text { bottom material }\end{array}$ & 6 & .00 & .00 & .0 & .0 & .00 \\
\hline $\begin{array}{l}\text { Methoxychlor, total in } \\
\text { bottom material }\end{array}$ & 6 & .00 & .00 & .0 & .0 & .00 \\
\hline $\begin{array}{l}\text { Mirex, total in } \\
\text { bottom material }\end{array}$ & 4 & .00 & .00 & .0 & .0 & .00 \\
\hline $\begin{array}{l}\text { PCB, total in } \\
\text { bottom material }\end{array}$ & 6 & 23.2 & 25.2 & 4.0 & 68 & 10.3 \\
\hline $\begin{array}{l}\text { PCN, total in } \\
\text { bottom material }\end{array}$ & 4 & .00 & .00 & .0 & .0 & .00 \\
\hline $\begin{array}{l}\text { Perthane, total in } \\
\text { bottom material }\end{array}$ & 4 & .00 & .00 & .0 & .0 & .00 \\
\hline $\begin{array}{l}\text { Toxaphene, total in } \\
\text { bottom material }\end{array}$ & 6 & .00 & .00 & .0 & .0 & .00 \\
\hline
\end{tabular}


Table 7.-Summary of measurements of constituents in the bottom material for each station (continued)

\begin{tabular}{|c|c|c|c|c|c|c|}
\hline Constituent & $\begin{array}{l}\text { Number } \\
\text { of } \\
\text { samples }\end{array}$ & Mean & $\begin{array}{l}\text { Standard } \\
\text { deviation }\end{array}$ & $\begin{array}{l}\text { Minimum } \\
\text { value }\end{array}$ & $\begin{array}{l}\text { Maximum } \\
\text { value }\end{array}$ & $\begin{array}{l}\text { Standard } \\
\text { error of } \\
\text { mean }\end{array}$ \\
\hline
\end{tabular}

01116617 Pawtuxet River at Pawtuxet, R. I.

(The-following analyses are in micrograms per gram except as indicated.)

C.O.D., total in bottom material $(\mathrm{mg} / \mathrm{kg})$

Aluminum, recoverable from bottom material

Arsenic, total in bottom material

Barium, recoverable from bottom material

Beryllium, recoverable from bottom material

Boron, recoverable from bottom material

Cadmium, recoverable from bottom material

Chromium, recoverable from bottom material

Cobalt, recoverable from bottom material

Copper, recoverable from bottom material

Iron, recoverable from bottom material

Lead, recoverable from bottom material

Manganese, recoverable from bottom material

Mercury, recoverable from bottom material

Molybdenum, recoverable from bottom material

Nickel, recoverable from bottom material

Selenium, total in bottom material

Silver, recoverable from bottom material

Strontium, recoverable from bottom material

Vanadium, total in bottom material

Zinc, recoverable from bottom material

11

11

$\mathbf{5 8 , 3 0 0} \quad \mathbf{9 5 , 7 0 0}$

2

4,550

2,190

4,900

330,000

28,900

7

.1

.4

3,000

6,100

1,550

2

110

99

0

1

.1

2

1.0

1.4

40

180

70

5

4.0

8.9

0

2

1.0

7

7

1.6

2.7

0

20

4.0

7

35.7

27.0

0

6

1.0

7

7

.0

.0

10

90

10.2

7

116.9

150

0

0

.0

7

5,560

4,310

30

450

56.8

7

7

69.4

67.0

2,400

15,000

1,630

7

81.6

68.7

20

210

25.3

7

.01

32

230

26.0

.03

.0

.1

.01

2

.0

0

o

.0

7

17.3

17.1

0

50

6.5

7

.0

.0

0

0

.0

6

1.0

1.5

0

3

.6

2

25.0

21.2

10

40

15.0

0

7

228
343
40

1,000 
Table 7.-Summary of measure ments of constituents in the bottom material for each station (continued)

Constituent $\quad \begin{gathered}\text { Number } \\ \text { of } \\ \text { samples }\end{gathered}$ Mean $\quad \begin{gathered}\text { Standard } \\ \text { deviation }\end{gathered} \quad \begin{gathered}\text { Minimum value } \\ \text { vaximum } \\ \text { value }\end{gathered} \begin{gathered}\text { Standard } \\ \text { mean of } \\ \text { mean }\end{gathered}$

01116617 Pawtuxet River at Pawtuxet, R. I. (continued)

(The following analyses are in micrograms per kilogram.)

\begin{tabular}{|c|c|c|c|c|c|c|}
\hline $\begin{array}{l}\text { Aldrin, total in } \\
\text { bottom material }\end{array}$ & 6 & 0.00 & 0.00 & 0.0 & 0.0 & 0.00 \\
\hline $\begin{array}{l}\text { Chlordane, total in } \\
\text { bottom material }\end{array}$ & 6 & 31.2 & 46.6 & .0 & 110 & 19.0 \\
\hline $\begin{array}{l}\mathrm{DDD}, \text { total in } \\
\text { bottom material }\end{array}$ & 6 & 32.9 & 63.0 & .0 & 160 & 25.7 \\
\hline $\begin{array}{l}\mathrm{DDE}, \text { total in } \\
\text { bottom material }\end{array}$ & 6 & .55 & .88 & .0 & 2.0 & .36 \\
\hline $\begin{array}{l}\mathrm{DDT}, \text { total in } \\
\text { bottom material }\end{array}$ & 6 & 3.42 & 7.64 & .0 & 19 & 3.12 \\
\hline $\begin{array}{l}\text { Dieldrin, total in } \\
\text { bottom material }\end{array}$ & 6 & 5.57 & 8.94 & .0 & 22 & 3.65 \\
\hline $\begin{array}{l}\text { Endosulfan, total in } \\
\text { bottom material }\end{array}$ & 3 & 3.67 & 6.35 & .0 & 11 & $\mathbf{3 . 6 7}$ \\
\hline $\begin{array}{l}\text { Endrin, total in } \\
\text { bottom material }\end{array}$ & 6 & 1.25 & 3.06 & .0 & 7.5 & 1.25 \\
\hline $\begin{array}{l}\text { Heptachlor, total in } \\
\text { bottom material }\end{array}$ & 6 & .08 & .20 & .0 & .5 & .08 \\
\hline $\begin{array}{l}\text { Heptachlor epoxide, total } \\
\text { in bottom material }\end{array}$ & 6 & .00 & .00 & .0 & .0 & .00 \\
\hline $\begin{array}{l}\text { Lindane, total in } \\
\text { bot tom material }\end{array}$ & 6 & .00 & .00 & .0 & .0 & .00 \\
\hline $\begin{array}{l}\text { Methoxychlor, total in } \\
\text { bottom material }\end{array}$ & 6 & .00 & .00 & .0 & .0 & .00 \\
\hline $\begin{array}{l}\text { Mirex, total in } \\
\text { bottom material }\end{array}$ & 3 & .00 & .00 & .0 & .0 & .00 \\
\hline $\begin{array}{l}\text { PCB, total in } \\
\text { bottom material }\end{array}$ & 6 & 986 & 2,160 & 19 & 5,400 & 884 \\
\hline $\begin{array}{l}\text { PCN, total in } \\
\text { bottom material }\end{array}$ & 3 & .00 & .00 & .0 & .0 & .00 \\
\hline $\begin{array}{l}\text { Perthane, total in } \\
\text { bottom material }\end{array}$ & 3 & .00 & .00 & .0 & .0 & .00 \\
\hline $\begin{array}{l}\text { Toxaphene, total in } \\
\text { bottom material }\end{array}$ & 6 & .00 & .00 & .0 & .0 & .00 \\
\hline
\end{tabular}


Table 7.-Summary of measurements of constituents in the bottom material for each station (continued)

\begin{tabular}{|c|c|c|c|c|c|c|}
\hline Constituent & $\begin{array}{l}\text { Number } \\
\text { of } \\
\text { samples }\end{array}$ & Mean & $\begin{array}{l}\text { Standard } \\
\text { deviation }\end{array}$ & $\begin{array}{l}\text { Minimum } \\
\text { value }\end{array}$ & $\begin{array}{l}\text { Maximum } \\
\text { value }\end{array}$ & $\begin{array}{l}\text { Standard } \\
\text { error of } \\
\text { mean }\end{array}$ \\
\hline \multicolumn{3}{|c|}{ (The following analyses are in micrograms per gram except as indicated.) } & $\begin{array}{l}\text { er at Weste } \\
\text { ms per gram }\end{array}$ & $\begin{array}{l}\text {, R. I. } \\
\text { xcept as inc }\end{array}$ & cated.) & \\
\hline $\begin{array}{l}\text { C.O.D., total in bottom } \\
\text { material (mg/kg) }\end{array}$ & 12 & 19,600 & 25,900 & 2,800 & 96,000 & 7,490 \\
\hline $\begin{array}{l}\text { Aluminum, recoverable from } \\
\text { bottom material }\end{array}$ & 2 & 1,450 & 212 & 1,300 & 1,600 & 150 \\
\hline $\begin{array}{l}\text { Arsenic, total in } \\
\text { bottom material }\end{array}$ & 5 & .0 & .0 & 0 & 0 & .0 \\
\hline $\begin{array}{l}\text { Barium, recoverable from } \\
\text { bottom material }\end{array}$ & 2 & 10 & 14 & 0 & 20 & 10 \\
\hline $\begin{array}{l}\text { Beryllium, recoverable from } \\
\text { bottom material }\end{array}$ & 2 & .0 & .0 & 0 & 0 & .0 \\
\hline $\begin{array}{l}\text { Boron, recoverable from } \\
\text { bottom material }\end{array}$ & 2 & .0 & .0 & 0 & 0 & .0 \\
\hline $\begin{array}{l}\text { Cadmium, recoverable from } \\
\text { bottom material }\end{array}$ & 5 & .0 & .0 & $\mathbf{0}$ & 0 & .0 \\
\hline $\begin{array}{l}\text { Chromium, recoverable from } \\
\text { bottom material }\end{array}$ & 5 & 6.0 & 5.5 & 0 & 10 & 2.5 \\
\hline $\begin{array}{l}\text { Cobalt, recoverable from } \\
\text { bottom material }\end{array}$ & 5 & .0 & .0 & 0 & 0 & .0 \\
\hline $\begin{array}{l}\text { Copper, recoverable from } \\
\text { bottom material }\end{array}$ & 5 & 4.6 & 8.7 & 0 & 20 & 3.9 \\
\hline $\begin{array}{l}\text { Iron, recoverable from } \\
\text { bottom material }\end{array}$ & 5 & 1,750 & 890 & 740 & 3,100 & 398 \\
\hline $\begin{array}{l}\text { Lead, recoverable from } \\
\text { bottom material }\end{array}$ & 5 & 98.4 & 213 & 0 & 480 & 95.4 \\
\hline $\begin{array}{l}\text { Manganese, recoverable from } \\
\text { bottom material }\end{array}$ & 5 & 88.2 & 109 & 13 & 280 & 48.8 \\
\hline $\begin{array}{l}\text { Mercury, recoverable from } \\
\text { bottom material }\end{array}$ & 5 & .10 & .23 & .0 & .5 & .10 \\
\hline $\begin{array}{l}\text { Molybdenum, recoverable from } \\
\text { bottom material }\end{array}$ & 2 & .0 & .0 & 0 & 0 & .0 \\
\hline $\begin{array}{l}\text { Nickel, recoverable from } \\
\text { bottom material }\end{array}$ & 4 & 1.2 & 2.5 & 0 & 5 & 1.2 \\
\hline $\begin{array}{l}\text { Selenium, total in } \\
\text { bottom material }\end{array}$ & 5 & .0 & .0 & 0 & 0 & .0 \\
\hline $\begin{array}{l}\text { Silver, recoverable from } \\
\text { bottom material }\end{array}$ & 6 & .0 & .0 & 0 & 0 & .0 \\
\hline $\begin{array}{l}\text { Strontium, recoverable from } \\
\text { bottom material }\end{array}$ & 2 & 15.0 & 7.1 & 10 & 20 & 5.0 \\
\hline $\begin{array}{l}\text { Vanadium, total in } \\
\text { bottom material }\end{array}$ & 0 & - & - & - & - & - \\
\hline $\begin{array}{l}\text { Zine, recoverable from } \\
\text { bottom material }\end{array}$ & 5 & 13.8 & 9.1 & 8 & 30 & 4.1 \\
\hline
\end{tabular}


Table 7.-Summary of measurements of constituents in the bottom material for each station (continued)

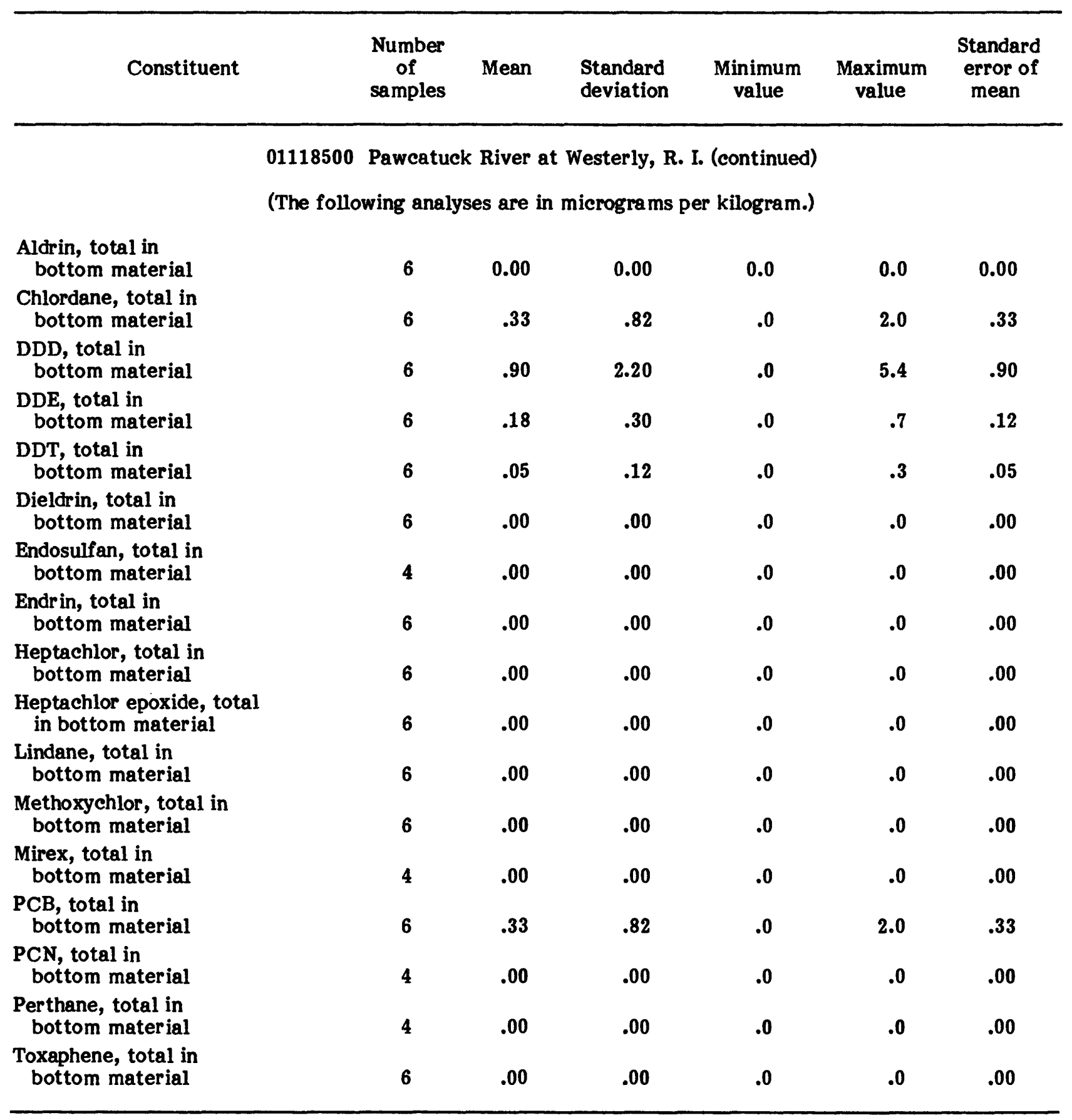




\section{TRENDS IN WATER QUALITY}

One of the most frequently asked questions, "Is water quality getting better or worse?", is one of the most difficult questions to answer. The very nature of water quality means that over a period of time there will be a considerable variability in the constituent concentrations. How then is one able to look at the record of analytical data and decide if there is a change in the concentration of the constituent? The simplest way to look for a change is to compare the data over time. Is a given constituent increasing or decreasing? Water quality constituents, such as dissolved solids, are difficult to compare over time because they often are related to the flow of water in the stream. For example in a particular river, dissolved solids concentration may decrease with an increase in flow and increase as the flow diminishes. If one examines the record of dissolved solids over time there may, for example, seem to be a decrease over the period of record in dissolved solids concentration. In this case, the record may have begun during a dry period when streamflow was below normal and ended during a wet period with streamflow above normal. There is a trend but it is related to the flow of water in the stream. While this information may be useful, the primary concern of most water managers and the public is to know if the efforts to clean up water pollution sources are having a positive effect.

The seasonality of the data also is important. For example, streamflows are commonly lowest in the late summer and early fall. If a water-quality constituent varies with streamflow then the constituent will be at a higher (or lower) concentration during the late summer and early fall. Conversely, at times of the year when streamflow is high, the concentration will be at a lower (or higher) level.

Other problems in trend detection include the skewness of the data and the serial correlation of the data. To avoid all of these problems, Hirsch and others (1982, p. 5-6) derived a modification of Kendall's Tau test known as the Seasonal Kendall test. Smith and others (1982) briefly describe the test as:

"The null hypothesis for this test is that the random variable is independent of time. The only necessary background assumption is that the random variable is independent and identically distributed (with any distribution). In this test, all possible pairs of data values are compared; if the later value (in time) is higher, a plus is scored; if the later value is lower, a minus is scored. If there is no trend in the data, the odds are 50-50 that a value is higher (or lower) than one of its predecessors. In the absence of a trend, the number of pluses should be about the same as the number of minuses. If, however, there are many more pluses than minuses, the values later in the series are more frequently higher than those earlier in the series, and so an uptrend is likely. Similarly, if there are many more minuses than pluses, a downtrend is likely.

"As discussed above, the one common pattern to water-quality variables is that they have a period of one year (other periodicities may exist). Comparing, for example, a January value with a May value does not contribute any information about the existence of a trend, if a seasonal cycle of a 1-year period exists. Thus, we define the Seasonal Kendall test to be the Kendall's Tau test resticted to those pairs of data which are multiples of 12 months apart. Since comparision are made only between data from the same month of the year, the problem of seasonality is avoided. Thus, the background assumptions given above are relaxed. The random variable may be nonidentically distributed, provided that the distributions 12 months apart are identical." 


\section{Method}

Table 8 shows the results of the Seasonal Kendall test using total phophorus, total nitrogen, and specific conductance at the six sites. The methods used are fully described in Crawford and others (1983) and Smith and others (1982). Briefly the first step is to determine if the concentration of the constituent is related to the discharge. Specific conductance, while not a concentration value, has been treated as one for this analysis. A series of 14 different relationships (Crawford and others, 1983) was tested for each of the constituents at each of the six sites. However, if a relationship existed at the site, either of the following best fit the data:

(1) $C=a+b(Q) \quad$ linear

(2) $C=a+b \ln (Q) \quad \log$-linear

Where $\mathrm{C}$ is the predicted concentration, $\mathrm{Q}$ is the discharge, in cubic feet per second, and $a$ and $b$ are constants.

In table 8, the type of relationship is given under the heading "Flow-adjusted concentration", along with the slope of the equation (whether $b$ is positive or negative) for each station and constituent. These equations are labeled with "hs", highly significant, and $" s$ " significant, depending on the $p$ value where $p$ is the probability of erroneously rejecting the null hypothesis that $b=0$; that is, there is no relationship with discharge. The regression type was marked "highly significant" where $p$ is less than or equal to 0.01 , and significant where $p$ is less than or equal to 0.1 . While regression $p$ values provide an appropriate basis for deciding when to make adjustments for flow dependency (Smith and others, 1982) a measure of the predictability of the concentration of the constituent based on flow is given by the proportion of variance explained, $R^{2}$ ( $R$ squared in the table). The closer $R^{2}$ is to 1.0 , the better the equation fits the data.

A negative discharge-constituent relationship, which is the case for all three constituents and all sites, generally indicates that dilution either of point-source contributions or of subsurface sources is the dominant process. Smith and others (1982) found that this negative relationship for total phosphorus is generally limited to forested basins along the east coast, the Great Lakes, and the California coast. Nationwide, the majority of rivers have a positive discharge-total phosphorus relationship indicating that erosion and transport of total phophorus at high flows is the dominant process.

After the discharge-constituent relationship was determined, the next step was to run the Seasonal Kendall test on the discharge, concentration, transport, and the flowadjusted concentration. Transport, which was computed for total nitrogen and phophorus but not for specific conductance, is the product of the concentration and the discharge

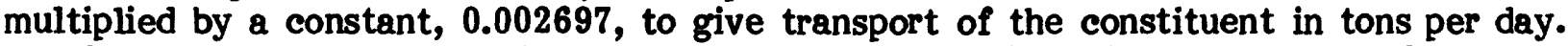
The flow-adjusted concentration is the actual concentration minus the expected concentration for that particular analysis calculated using one of the equations given above and indicated on the table.

\section{$\underline{\text { Results }}$}

As Smith and others (1982) point out, trend analyses "...will never reveal the cause of a change in stream quality, but they can lead to improved understanding of the kinds of causes to look for." Trends in discharge indicated what has happened to the amount of water in the river during the period of record. Generally this may be related to natural variations of flow based on the variations in rainfall. However, if diversion of water from the basin were begun or increased during the period of record, this would reveal a trend in discharge. Trends in concentration indicate what has happened over the period of record to the concentration. This can be of importance if the concentration is close 
Table 8.-Trend results for each station

(Trends and regressions are marked with "hs" for highly significant when $p$ is less than or equal to 0.01 , and with " 8 " for significant when $p$ is less than or equal to 0.1 . Slope is marked with a plus $(+)$ for a positive discharge-constituent relationship, and with a minus $(-)$ for a negative relationship. The term, "Trend (percent per year)", is the slope as milligrams per liter, tons per day, or micromhos, per year divided by the average value in milligrams per liter (tons per day or micromhos) and multiplied by 100 . The units in all cases are percent of mean per year.)

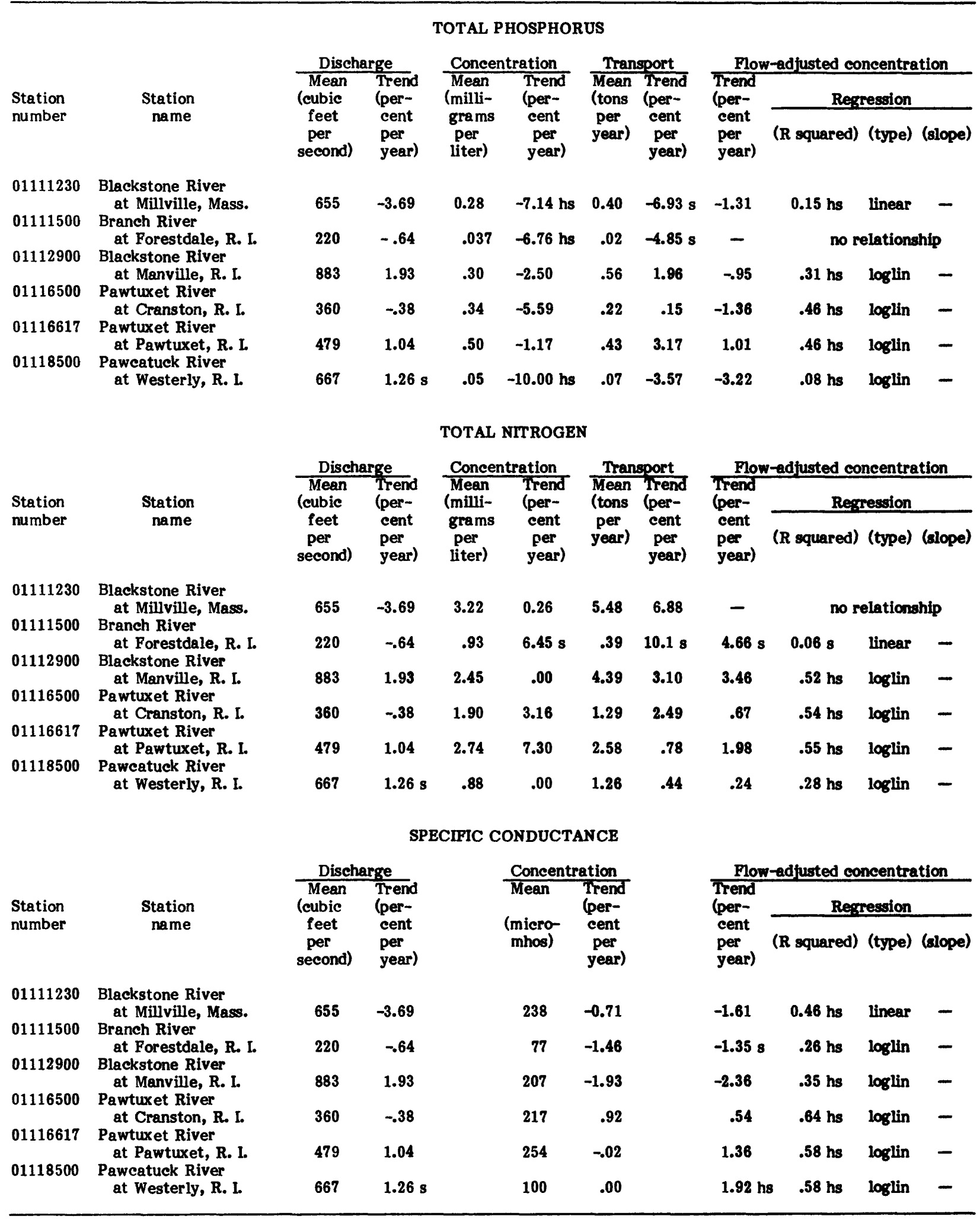


to an established criteria or standard. Trends in transport indicate what changes have occurred in the flux of substances through the river system, suggesting what might be happening to the rates of output from various sources of the constituent. For example, if a new wastewater treatment plant were installed which removed a significant part of the phosphorus formerly introduced into the stream, a trend in the transport would be detected. This trend might also be seen in the concentration but it might be masked by the variations in flow. The flow-adjusted concentration, which is the actual concentration minus the expected concentration using the discharge-constituent relationship, also should show a downward trend. If the processes other than flow which contribute the constituent to the river have not changed during the period of record, the flow-adjusted concentration will fluctuate randomly about zero. If the flow conditions change during the period of record they will be compensated for and will not affect the determination of a trend. Trends in the flow-adjusted concentration indicate that changes have occurred in the processes that deliver the constituent to the river.

Table 8 shows the results of the Seasonal Kendall test. The trend values are shown in percentage terms for ease of comparison. The term, "Trend (percent per year)", is the slope as milligrams per liter, tons per day, or micromhos, per year divided by the average value in milligrams per liter (tons per day or micromhos) and multiplied by 100 . The units in all cases are percent of mean per year. For the flow-adjusted concentration, the units are percent of mean concentration per year. Those slopes that are statistically significant at the 10 percent level are marked with an "s" and those at the 1 percent level by an "hs", corresponding to significant and highly significant, respectively.

Results for total phosphorus show significant downward trends in concentration and transport at the Blackstone River at Millville, Mass. However, neither the discharge nor the flow-adjusted concentration show a significant trend. Neither nitrogen or specific conductance showed any significant trends at the station.

Branch River at Forestdale also showed significant downward trends in phosphorus concentration and transport. Total nitrogen, however, showed a significant upward trend in concentration, transport, and flow-adjusted concentration. Specific conductance showed a significant downward trend for flow-adjusted concentration.

The only other site that showed any significant trends was the Pawcatuck River at Westerly. This site showed an upward trend in discharge, a downward trend in total phosphorus concentration, and an upward trend in flow-adjusted concentration for specific conductance.

\section{SUMMARY}

Concentrations of the common constituents were low at all Rhode Island stream sites between November 1979 and September 1983. The mean hardness at all sites was in the "soft" category although the maximum hardness might be in the moderately hard range. Mean sodium values met the most stringent drinking water criterion of $20 \mathrm{mg} / \mathrm{L}$ at the Branch River at Forestdale and the Pawcatuck River at Westerly. Mean concentrations at the other sites were slightly higher.

Nitrogen concentrations (total nitrate plus nitrite and total nitrogen) were lowest in the Branch River at Forestdale and the Pawcatuck River at Westerly while the mean total nitrate plus nitrite concentrations for the Blackstone River at Millville, Mass., and the Blackstone River at Manville were in the range that could cause undesirable growth of aquatic plants. Total phosphorus values were also lowest on the Branch River at Forestdale and the Pawcatuck River at Westerly. At the other sites, mean total phosphorus values were sufficiently high to potentially allow undesirable growth of aquatic plants if the waters were impounded.

Fecal coliform bacteria counts were high at all sites which indicated that the sites would not have met the criterion for body contact sports. Bacteria samples were not collected with sufficent frequency to indicate if the requirements of the criterion were met. 
Trace element concentrations in the stream were generally low. Those trace elements which were found in concentrations near or exceeding any standard or criterion include cadmium, chromium, lead, iron, and manganese. Cadmium concentrations were found at the drinking water standard of $10 \mu \mathrm{g} / \mathrm{L}$ at the Blackstone River at Millville, Mass., and the Pawcatuck River at Westerly. A total chromium concentration was at the drinking water standard of $50 \mu \mathrm{g} / \mathrm{L}$ at one site, the Blackstone River at Millville, Mass. Lead concentrations exceeded the drinking water standard of $50 \mu \mathrm{g} / \mathrm{L}$ at the Blackstone River at Millville, Mass. Manganese concentrations exceeded the drinking water standard at all sites and iron at several sites. High concentrations of trace elements in bottom materials were found at several sites including the Blackstone River at Millville, Mass.

Several pesticides and other organic compounds were found at the sites. Lindane, dieldrin, and PCB were found in the water. Phenols were found at all of the sites in excess of the $1 \mu \mathrm{g} / \mathrm{L}$ criterion for domestic water supplies and to protect against fish flesh tainting. Other organic compounds found in the bottom material samples, included aldrin, chlordane, DDD, DDE, DDT, dieldren, endosulfan, endrin, heptachlor, mirex, and PCB. One bottom material sample from the Pawtuxet River at Pawtuxet had a PCB concentration of $5400 \mu \mathrm{g} / \mathrm{kg}$.

Results of trend analysis of total phosphorus, total nitrogen, and specific conductance show a downward trend in phosphorus at Blackstone River at Millville, Mass., and Branch River at Forestdale. The Branch River at Forestdale had an upward trend in nitrogen. Specific conductance showed a downward trend at Branch River at Forestdale and an upward trend at Pawcatuck River at Westerly.

\section{REFERENCES CITED}

Briggs, J. C., and Ficke, J. F., 1978, Quality of rivers of the United States, 1975 water year-based on the National Stream Quality Accounting Network (NASQAN): U.S. Geological Survey Open-File Report 78-200, 436 p.

Crawford, C. G., Slack, J. R., and Hirsch, R. M., 1983, Nonparametric tests for trends in water-quality data using the Statistical Analysis System: U.S. Geological Survey Open-File Report 83-550, 102 p.

Durfor, C. N., and Becker, Edith, 1964, Public water supplies of the 100 largest cities in the United States, 1962: U.S. Geological Survey Water-Supply Paper 1812, 364 p.

Greeson, P. E., ed., 1979, A supplement to-Methods for collection and analysis of aquatic biological and microbiological samples (U.S. Geological Survey Techniques of Water-Resources Investigations, book 5, chap. A4): U.S. Geological Survey OpenFile Report 79-1279, 92 p.

Greeson, P. E., Ehlke, T. A., Irwin, G. A., Lium B. W., and Slack, K. V., eds., 1977, Methods for collection and analysis of aquatic biological and microbiological samples: U.S. Geological Survey Techniques of Water-Resources Investigations, book 5, chap. A4, 332 p.

Guy, H. P., and Norman, V. W., 1970, Field methods for measurement of fluvial sediment: U.S. Geological Survey Techniques of Water-Resources Investigations, book 3, chap. C2, 59 p.

Hirsch, R. M., Slack, J. R., and Smith, R. A., 1982, Techniques of trend analysis for monthly water quality data: Water Resources Research, v. 18, no. 1, p. 107-121. 
National Academy of Sciences and National Academy of Engineering, 1972 [1974], Water quality criteria 1972: Washington, D.C., U.S. Government Printing Office, Ecological Research Series, EPA-R3-73-033-March 1973, 594 p.

Rantz, S. E., and others, 1982, Measurement and computation of streamflow: U.S. Geological Survey Water-Supply Paper 2175, 631 p.

Skougstad, M. W., Fishman, M. J., Friedman, L. C., Erdmann, D. E., and Duncan, S. S., eds., 1979, Methods for determination of inorganic substances in water and fluvial sediments: U.S. Geological Survey Techniques of Water-Resources Investigations, book 5, chap. A1, 626 p.

Smith, R. A., Hirsch, R. M., and Slack, J. R., 1982, A study of trends in total phosphorus measurements at NASQAN stations: U.S. Geological Survey Water-Supply Paper 2190, 34 p.

U.S. Council on Environmental Quality, 1975, Environmental quality, 1975-Sixth annual report of the Council on Environmental Quality: Washington, D.C., U.S. Government Printing Office, $763 \mathrm{p}$.

1976, Environmental quality, 1976-Seventh annual report of the council on Environmental Quality: Washington, D.C., U.S. Government Printing Office, 378 p.

U.S. Environmental Protection Agency, 1975, National interim primary drinking water regulations: Federal Register, v.40, no. 248, Dec. 24, 1975, p. 59566-59588.

1976, Quality criteria for water: U.S. Environmental Protection Agency, 256 p.

1977, National secondary drinking water regulations: Federal Register, v. 42 , no. 62, March 31, 1977, p. 17143-17147.

1980, Interim primary drinking water regulations: Federal Register, v. 45, no. 168, Aug. 27, 1980, p. 57332-57357.

Wentz, D. A., 1974, Effect of mine drainage on the quality of streams in Colorado, 197172: Denver, Colorado Water Conservation Board, Colorado Water Resources Circular 21, 117 p., 3 pl.

Wershaw, R. L., Fishman, M. J., Grabbe, R. R., and Lowe, L. E., eds., 1983, Methods for the determination of organic substances in water and fluvial sediments: U.S. Geological Survey Techniques of Water-Resources Investigations, book 5, chap. A3, (released as Open-File Report 82-1004), 173 p.

Wood, W. W., 1976, Guidlines for collection and field analysis of ground-water samples for selected unstable constituents: U.S. Geological Survey Techniques of WaterResources Investigations, book 1, chap. D2, 24 p. 